\title{
Endogenous glucocorticoids prevent gastric metaplasia by suppressing spontaneous inflammation
}

\author{
Jonathan T. Busada, ${ }^{1}$ Sivapriya Ramamoorthy, ${ }^{1}$ Derek W. Cain, ${ }^{2}$ Xiaojiang Xu, ${ }^{3}$ Donald N. Cook, ${ }^{4}$ and John A. Cidlowski' \\ ${ }^{1}$ Molecular Endocrinology Group, Signal Transduction Laboratory, National Institute of Environmental Health Sciences, National Institutes of Health, Research Triangle Park, North Carolina, USA. ${ }^{2}$ Duke \\ Human Vaccine Institute, Duke University School of Medicine, Durham, North Carolina, USA. Integrative Bioinformatics Support Group and ${ }^{4}$ Immunogenetics Group, Immunity, Inflammation, and Disease \\ Laboratory, National Institute of Environmental Health Sciences, National Institutes of Health, Research Triangle Park, North Carolina, USA
}

\begin{abstract}
In the stomach, chronic inflammation causes metaplasia and creates a favorable environment for the evolution of gastric cancer. Glucocorticoids are steroid hormones that repress proinflammatory stimuli, but their role in the stomach is unknown. In this study, we show that endogenous glucocorticoids are required to maintain gastric homeostasis. Removal of circulating glucocorticoids in mice by adrenalectomy resulted in the rapid onset of spontaneous gastric inflammation, oxyntic atrophy, and spasmolytic polypeptide-expressing metaplasia (SPEM), a putative precursor of gastric cancer. SPEM and oxyntic atrophy occurred independently of lymphocytes. However, depletion of monocytes and macrophages by clodronate treatment or inhibition of gastric monocyte infiltration using the Cx3cr1 knockout mouse model prevented SPEM development. Our results highlight the requirement for endogenous glucocorticoid signaling within the stomach to prevent spontaneous gastric inflammation and metaplasia, and suggest that glucocorticoid deficiency may lead to gastric cancer development.
\end{abstract}

\section{Introduction}

Chronic inflammation is widely recognized as being a potent driver of cancer development and progression. Understanding the mechanisms that suppress pathogenic inflammation, and thereby cancer formation, is critical for the development of novel therapeutic strategies to treat cancer. Within the stomach, long-term Helicobacter pylori infection drives chronic inflammation leading to atrophic gastritis, oxyntic atrophy (parietal cell loss), and the development of potentially preneoplastic spasmolytic polypeptide-expressing metaplasia (SPEM) (1-3). Inflammation is a key driver of these pathological conditions and neoplasia is averted in Helicobacter-infected immune-deficient mouse models $(4,5)$. Several experimental models have shown that chronic inflammation is sufficient to induce gastric cancer development independent of $H$. pylori infection. Mice overexpressing the proinflammatory cytokines IL1B or IFNG in parietal cells, or that have dysregulated NFKB activity, exhibit spontaneous gastric inflammation leading to metaplasia and gastric cancer (6-8). In humans, polymorphisms in the $I L 1 B$ or NFKB1 genes are linked to gastric cancer development, even in the absence of $H$. pylori infection $(9,10)$. However, the molecular and cellular processes that regulate gastric inflammation and the mechanisms whereby the inflammatory milieu promotes metaplastic changes are poorly understood.

Glucocorticoids are steroid hormones secreted by the adrenal glands in response to an array of stimuli, including psychological and physiological stress, tissue damage, and inflammation (11).

Conflict of interest: The authors have declared that no conflict of interest exists. License: Copyright 2019, American Society for Clinical Investigation.

Submitted: July 3, 2018; Accepted: January 10, 2019.

Reference information: J Clin Invest. 2019;129(3):1345-1358.

https://doi.org/10.1172/JCl123233.
Among the best-studied role of glucocorticoids is their ability to modulate the immune system. Systemic adrenal insufficiency is tied to the development of chronic inflammatory and autoimmune disease (12). Glucocorticoids exert their antiinflammatory effects by binding to the glucocorticoid receptor (NR3C1, hereafter GR) which is a ligand-dependent transcription factor. Some of the functions of the GR are to suppress the transcription of proinflammatory cytokines and chemokines and to limit immune system activation in response to noxious stimuli (12). Synthetic glucocorticoids have been used extensively for over 50 years to treat a wide array of immune-related pathologies, and they remain one of the most effective therapies for treating inflammatory diseases of the gastrointestinal tract (13). Despite the widespread clinical use of synthetic glucocorticoids, the mechanisms whereby endogenous glucocorticoids modulate local immune activities during steady-state conditions are poorly understood. In the stomach, autoimmune and inflammatory gastritis are common symptoms of adrenal insufficiency $(14,15)$, and expression of the glucocorticoid receptor is disrupted in gastric cancer $(16,17)$. However, the functional requirement of endogenous glucocorticoid signaling to suppress gastric inflammatory disease is not known.

In this study, we tested the hypothesis that endogenous glucocorticoids play a critical role in suppressing gastric inflammation and maintaining gastric homeostasis. Endogenous corticosteroids were depleted in mice by bilateral adrenalectomy, resulting in chronic inflammation, oxyntic atrophy, and SPEM development within the lesser curvature of the gastric corpus. RNA sequencing of the gastric corpus 3 days after adrenalectomy revealed significant activation of proinflammatory pathways preceding leukocyte infiltration. Depletion of circulating monocytes/macrophages before adrenalectomy or inhibiting gastric infiltration of the CX3CR1 $1^{+}$monocyte subpopulation prevented SPEM develop- 
A

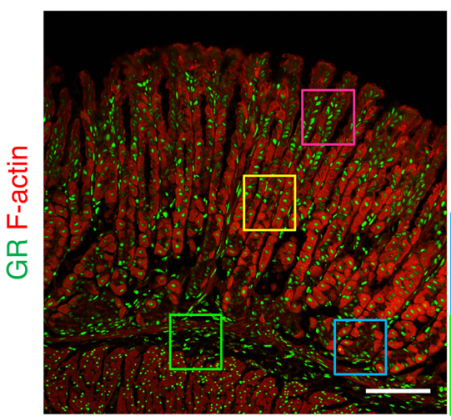

C

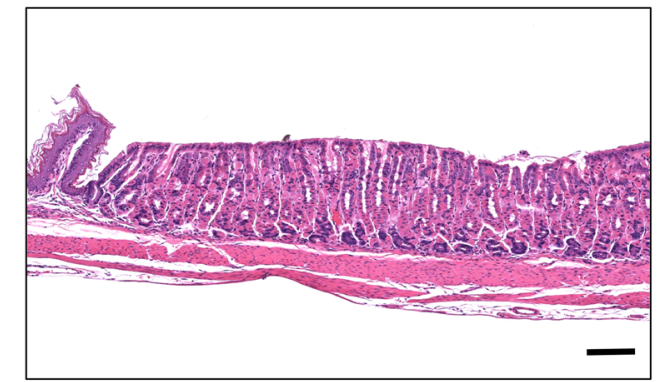

B

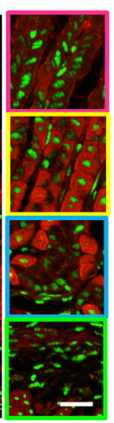

Sham-ADX

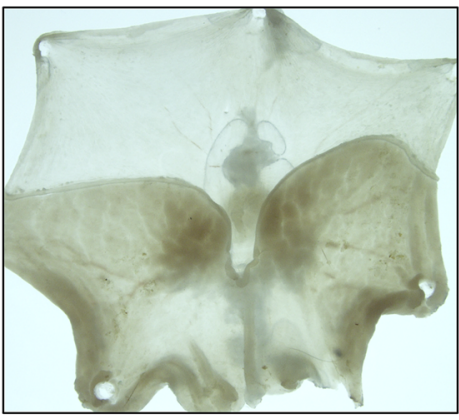

2 month ADX

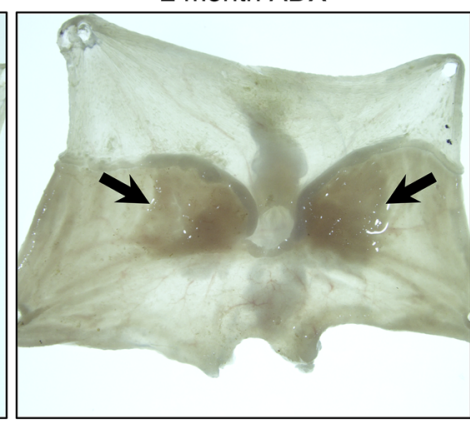

2 month ADX

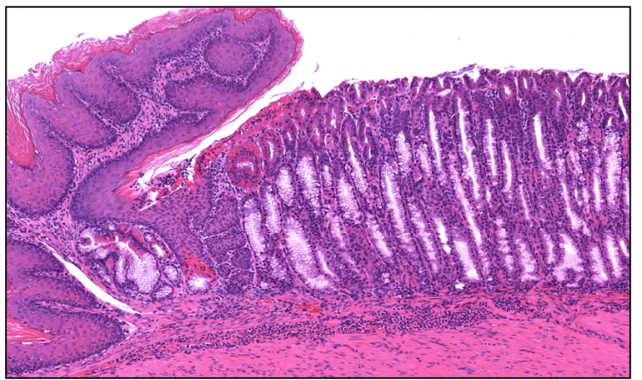

D

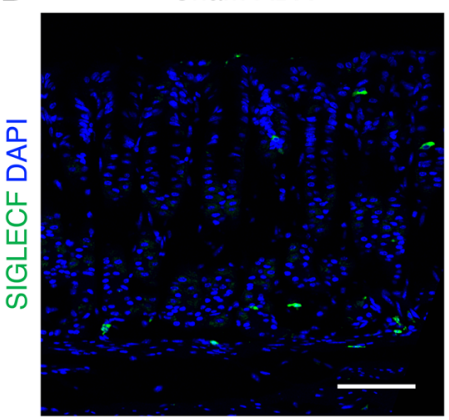

2 month $A D X$

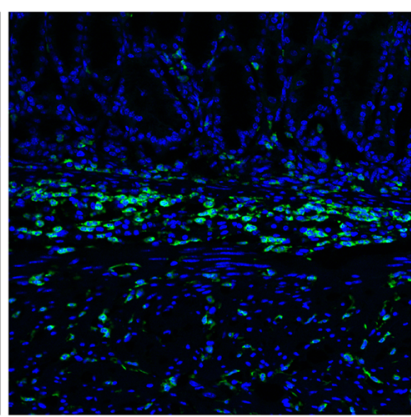

E

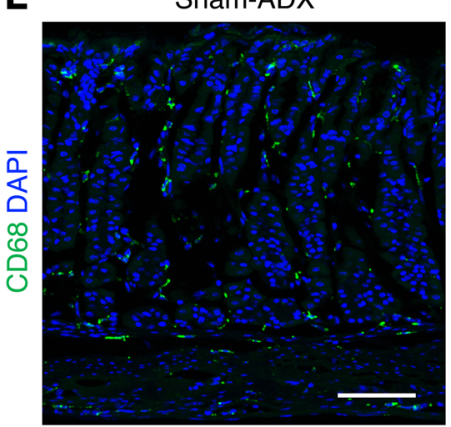

2 month ADX

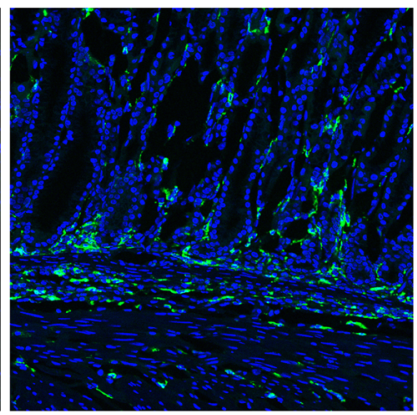

Figure 1. Adrenalectomy induces atrophic gastritis, mucous cell metaplasia, and chronic inflammation of the gastric corpus. Stomachs were collected from mice euthanized 2 months after sham surgery (Sham-ADX) or adrenalectomy (ADX). (A) Representative immunostaining of the gastric corpus lesser curvature probed for the glucocorticoid receptor (GR, green); F-actin was labeled with phalloidin (red). (B) Representative whole-mount images of mouse stomachs opened along the greater curvature. Arrows indicate the location of lesions, which develop after adrenalectomy. (C) Micrographs of H\&E-stained sections of the gastric corpus lesser curvature. (D-E) Immunostaining of stomach sections from the lesser curvature stained for SICLECF (eosinophils, green, D) or CD68 (macrophages, green, E). Nuclei were labeled with DAPI (blue). Scale bars: $100 \mu \mathrm{m}$ (inset in A: $25 \mu \mathrm{m}$ ); $n=8$ mice/group for all experiments.

ment. Our findings indicate that endogenous glucocorticoids are critical mediators of gastric homeostasis and provide the requisite signals to suppress spontaneous gastric inflammation. Thus, deficient glucocorticoid signaling may promote pathogenic gastric inflammation and gastric cancer evolution.

\section{Results}

Adrenalectomy induces atrophic gastritis and chronic inflammation of the gastric corpus. Glucocorticoid signaling through the GR provide the requisite signals to maintain homeostasis in a variety of tissues. However, the role of endogenous glucocorticoid signaling in the stomach remains unknown. We found that the GR is ubiquitously expressed throughout the mucosa and submucosa of the gastric corpus (Figure 1A and Supplemental Figure 1A; supplemental material available online with this article; https://doi. org/10.1172/JCI123233DS1). To investigate the requirement for endogenous glucocorticoids in the stomach, we performed bilateral adrenalectomy on WT C57BL/6J mice to deplete circulating corticosteroids (Supplemental Figure 1B). Circulating corticosterone, the endogenous mouse glucocorticoid, was maximally depleted by 3 days after adrenalectomy (Supplemental Figure 1C). Two months after adrenalectomy, there were conspicuous macroscopic lesions restricted to the gastric corpus lesser curvature adjacent to the esophagus (Figure 1B). Histological analysis of the lesion revealed that the corpus glands were enlarged and filled with mucous cells compared with sham-adrenalectomized controls (Figure 1C). In addition, there was conspicuous leukocyte infiltration throughout base of the gastric glands and adjacent submucosa. We used lineage-specific markers to characterize the immune-cell infiltrate. Immunostaining with the eosinophil marker SIGLECF and the monocyte/macrophage marker CD68 revealed a dramatic increase in these cell types 2 months after 
A

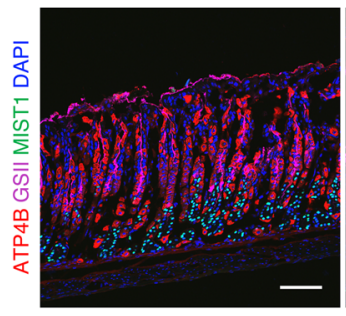

B

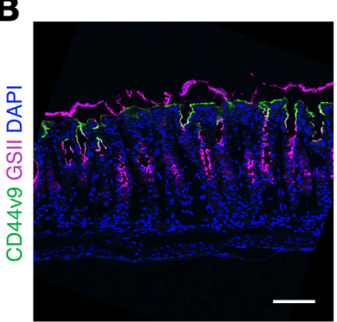

C
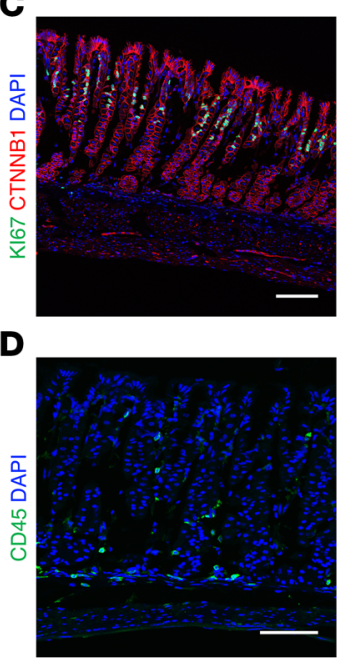

2 month $\mathrm{ADX}$
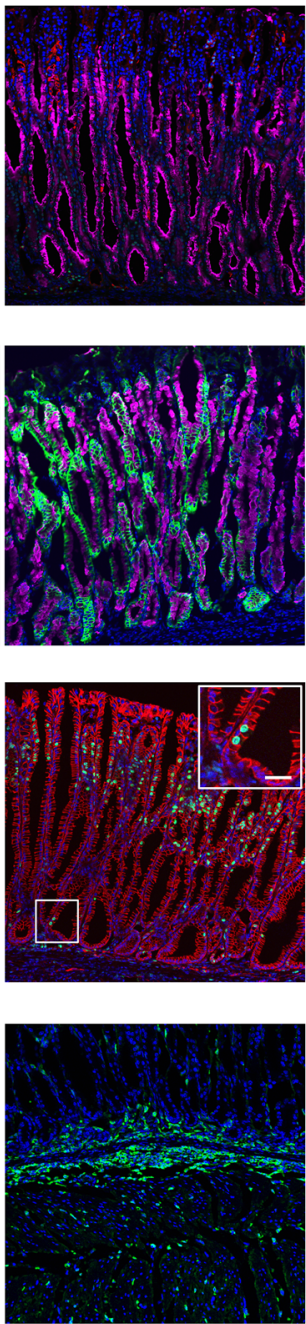

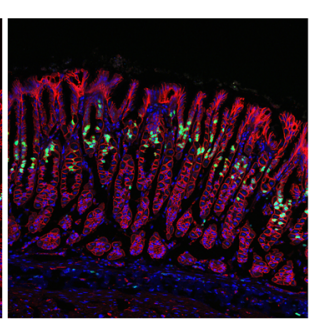

2 month ADX + Cort
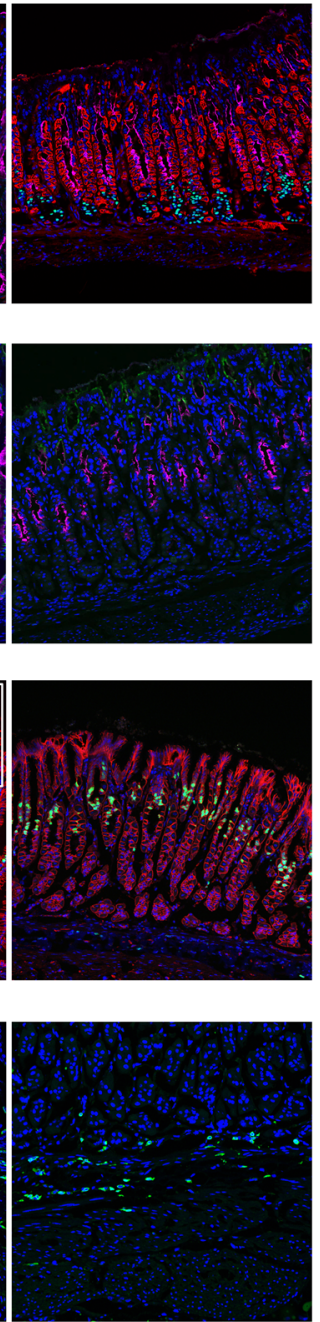

$\mathbf{E}$

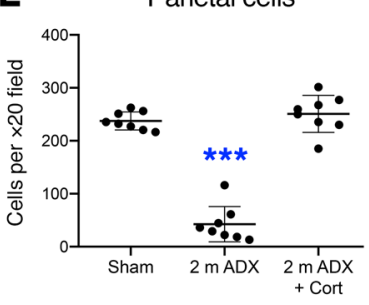

Chief cells
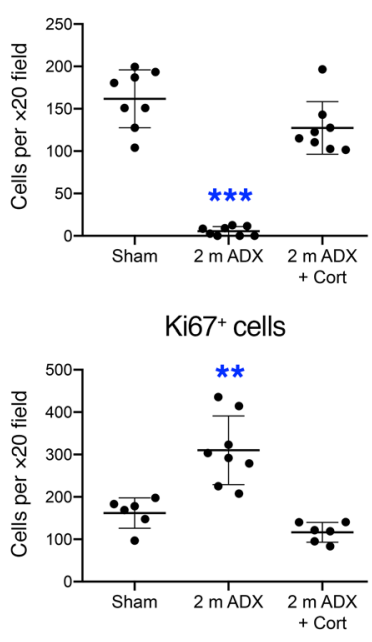

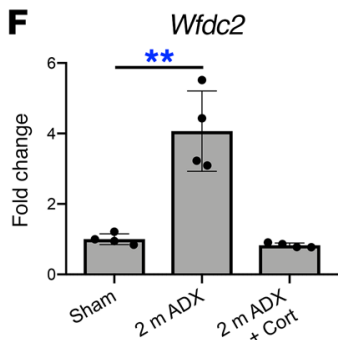

Olfm4
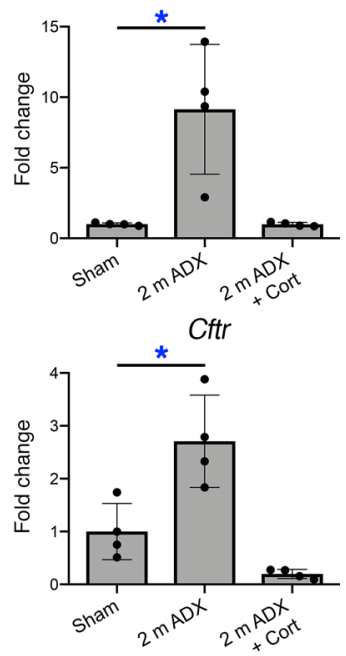

Figure 2. Endogenous glucocorticoids are required to suppress SPEM and gastric inflammation. Analysis of the gastric corpus lesser curvature from mice euthanized 2 months after sham surgery or adrenalectomy or from adrenalectomized mice treated with corticosterone (cort) for 2 months. (A-D) Immunostaining of stomach sections probed for (A) ATP4B (parietal cells, red), CSII lectin (mucous neck cells, pink), and MIST1(chief cells, green), (B) the SPEM marker CD44 variant 9 (green) and CSII lectin (pink), (C) KI67 (green) and CTNNB1 (epithelial cells, red), or for CD45 (leukocytes, green). Nuclei are stained with DAPI (blue). Scale bars: $100 \mu \mathrm{m}$ (inset in C: $25 \mu \mathrm{m}$ ). (E) Quantitation of the number of parietal cells, chief cells, and proliferating epithelial cells observed per $\times 20$ field within the lesser curvature ( $n \geq 6$ mice/group). (F) Quantitative RT-PCR of the SPEM marker genes: Wfdc2, Olfm4, and Cftr using RNA isolated from the gastric corpus lesser curvature ( $n=4$ mice/group). Data are mean $\pm \mathrm{SD} ; P$ values were determined by 1 -way ANOVA with post hoc Tukey's $t$ test. ${ }^{*} P \leq 0.01,{ }^{*} P \leq 0.001,{ }^{*}{ }^{*} P \leq 0.0001$.

adrenalectomy (Figure 1, D and E). Histological analysis revealed that adrenalectomy did not trigger gross morphological changes of the gastric pylorus (Supplemental Figure 1D).

Adrenal hormones are required to suppress SPEM development. To further analyze the changes that occur in the lesser curvature of the gastric corpus following adrenalectomy, we performed immunostaining for the parietal cell marker ATP4B, the mucous neck cellspecific (MNC-specific) lectin GSII, and the mature chief cell marker MIST1. Immunostaining revealed an $82 \%$ decrease in parietal cells and a $97 \%$ decrease in mature chief cells 2 months after adrenalectomy relative to sham controls (Figure 2, A and E). In sham animals, $\mathrm{GSII}^{+} \mathrm{MNCs}$ were restricted to the middle portion of the gastric glands and GSII did not colocalize with MIST1, but 2 months after adrenalectomy, GSII ${ }^{+}$cells were found throughout the entire gastric gland (Figure 2A). Gastric cells exhibit remarkable plasticity in response to damage. Within the corpus, injury triggers SPEM development through transdifferentiation of mature chief cells $(18,19)$. Acute SPEM is thought to promote wound healing $(20,21)$, but chronic inflammation and SPEM are associated with the development of gastric adenocarcinoma $(3,21)$. Loss of parietal cells, downregulation of the mature chief cell marker MIST1, and renewed proliferation of post-mitotic cells localized at the base of the gastric glands are hallmarks of SPEM (19). To examine the effects of adrenalectomy on SPEM development we performed immunostaining for the de novo SPEM marker genes CD44 variant 9 (CD44v9) and SOX9 (22-24). CD44v9 was not detectable in the gastric corpus of control animals, but was robustly induced and colocalized with GSII 2 months after adrenalectomy (Figure 2B). SOX9 was weakly expressed in the stomach of sham animals but was robustly induced throughout the gastric glands 2 months after adrenalectomy (Supplemental Figure 2). Adre- 
A

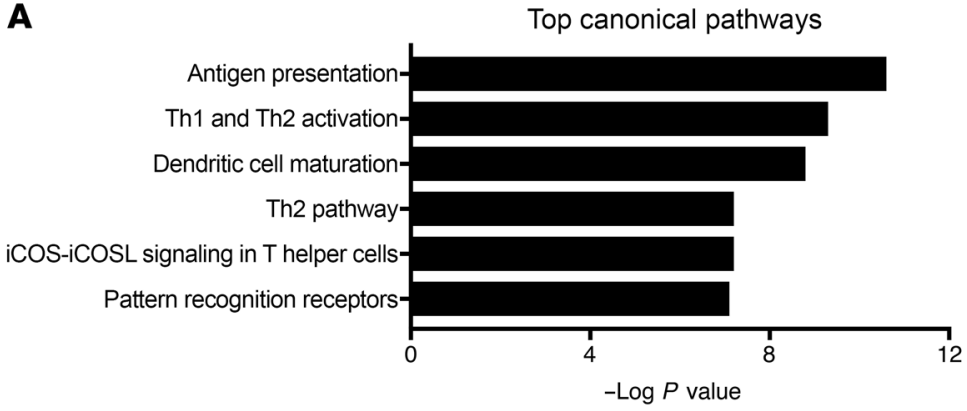

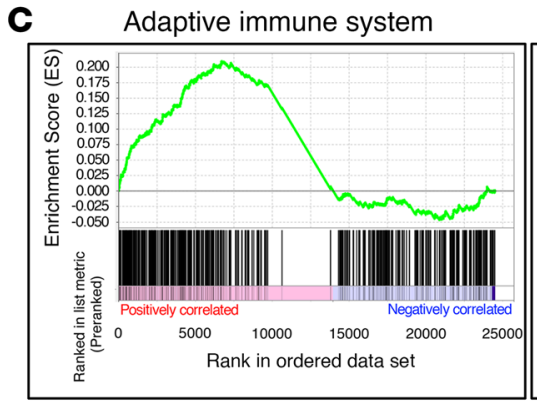

Cytokine signaling pathway

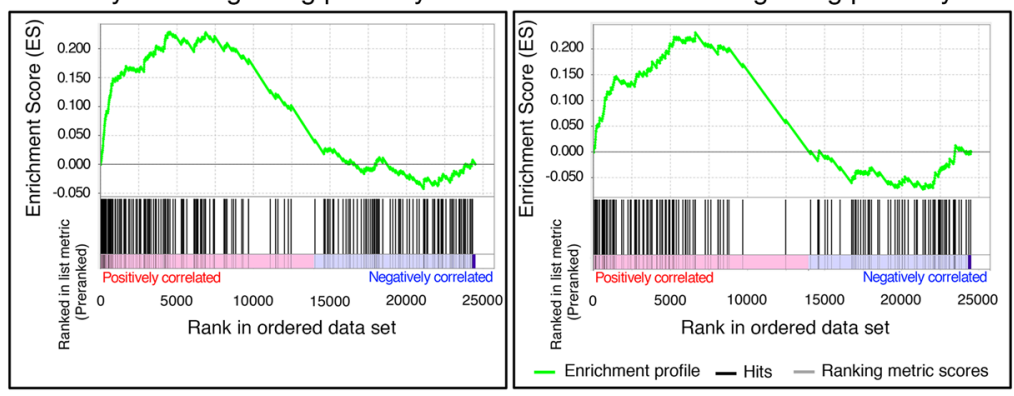

Innate immune system

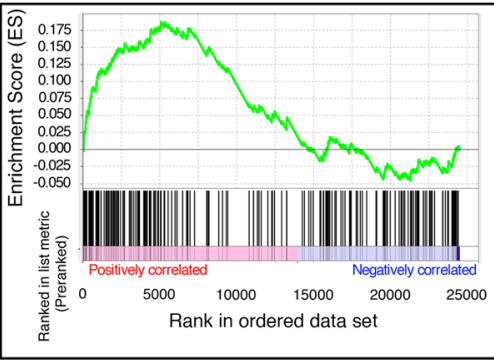

Chemokine signaling pathway
B
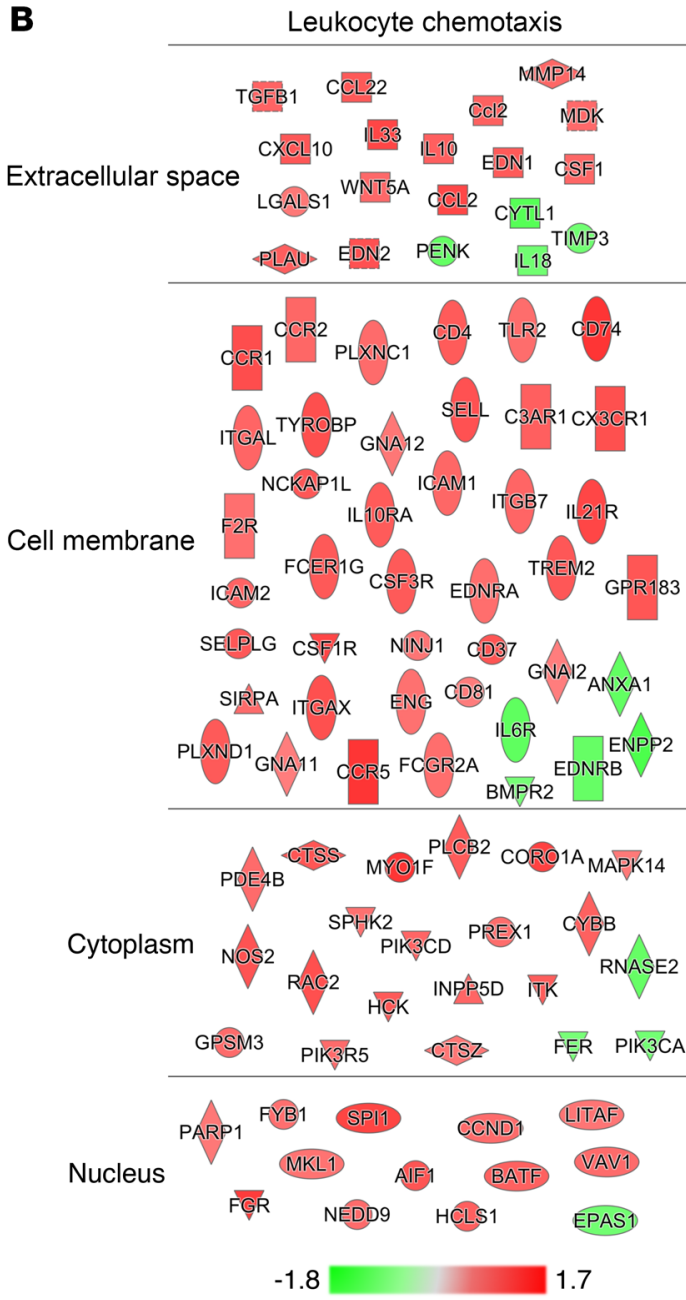

Figure 3. Endogenous glucocorticoids suppress spontaneous inflammation of the gastric mucosa. RNA sequencing of the gastric corpus lesser curvature from mice euthanized 3 days after sham surgery or adrenalectomy. (A) The top 6 canonical pathways identified by IPA ranked by $P$ value. (B) Induced (red) and repressed (green) genes associated with the leukocyte chemotaxis pathway identified by IPA. The scale bar represents fold change. (C) Gene set enrichment analysis. $n=4$ mice $/$ group.

nalectomy triggered a 2-fold increase in epithelial cell proliferation within the gastric corpus (Figure 2, C and E). While the bulk of $\mathrm{KI} 7^{+}$ cells were restricted to the gland isthmus, which is the normal proliferative compartment, adrenalectomized mice exhibited proliferating cells within the base of the gastric glands. SPEM development within the lesser curvature was further confirmed by real-time reverse transcription PCR (qRT-PCR) of the SPEM marker genes Wfdc2, Olfm4, and $\operatorname{Cftr}(25,26)$ which were significantly upregulated 4-fold, 9.1fold, and 2.7-fold, respectively, in adrenalectomized mice relative to sham controls (Figure 2F).

Within the gastric corpus, $H$. pylori infection disproportionately triggers inflammation, gastritis, and metaplasia in the lesser curvature $(3,27)$. Adrenalectomy-induced inflammation and gastritis followed a similar pattern and was predominately restricted to the lesser curvature whereas the greater curvature was histologically normal (Supplemental Figure 3A). Both regions of the corpus expressed similar levels of the GR, suggesting that differential response to adrenalectomy was not due to different requirements for glucocorticoid signaling (Supplemental Figure 1A). Moreover, immunostaining for CD68 revealed an equivalent number of macrophages in the greater curvature 2 months after adrenalectomy (Supplemental Figure 3B). Glucocorticoids have been reported to stimulate differentiation of parietal and chief cells in neonatal mice $(28,29)$. Adrenalectomy did not reduce the number of parietal cells within the greater curvature but did elicit a $43 \%$ decrease in mature chief cells (Supplemental Figure 3, C and D). The abundance of parietal cells and mature chief cells within the greater curvature suggests that depletion of these cell types within the lesser curvature is not a result of altered differentiation. CD44v9 was not detected in the corpus greater curvature 2 months after adrenalectomy (Supplemental Figure 3E).

Glucocorticoids are required to suppress gastric inflammation and SPEM. In addition to glucocorticoids, the adrenal glands produce the mineralocorticoid aldosterone and the catecholamines epinephrine and norepinephrine. To determine if glucocorticoids are the adrenal hormone required to maintain gastric homeostasis, mice were administered the endogenous mouse glucocorticoid corticosterone in drinking water for 2 months after adrenalectomy. Corticosterone replacement prevented the development of macroscopic lesions within the gastric corpus (Supplemental Figure 4A). Moreover, corticosterone 
A

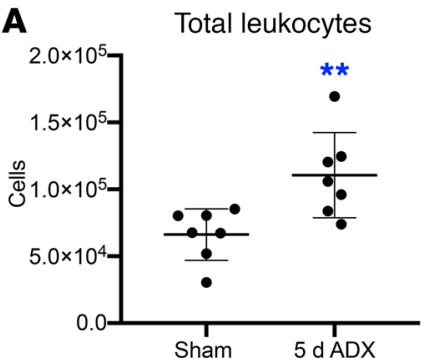

Eosinophils

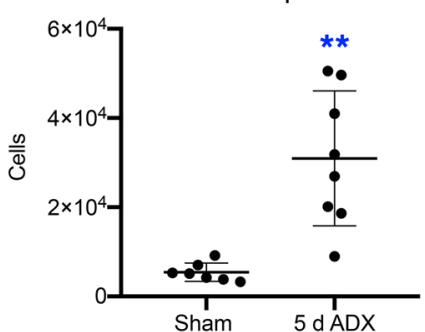

B
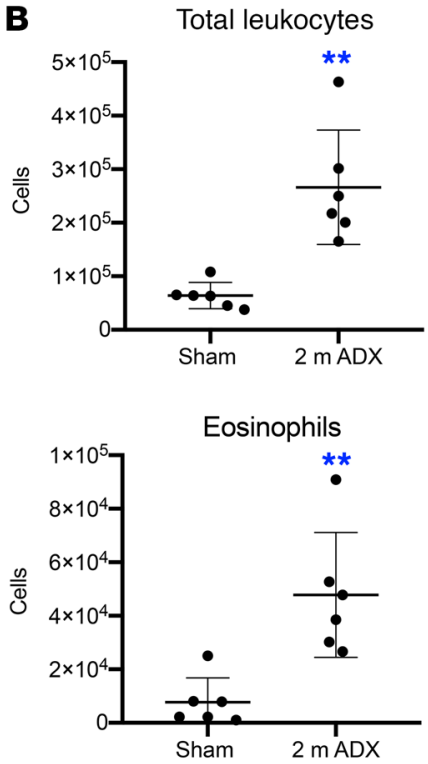

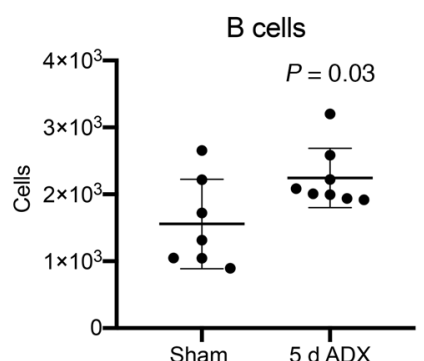

Macrophages

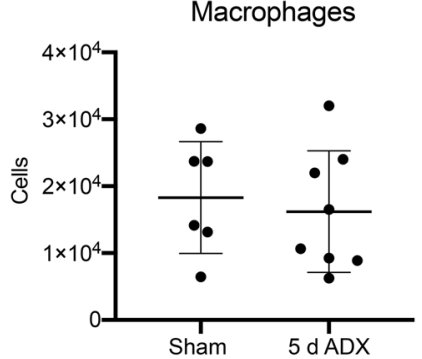

B cells

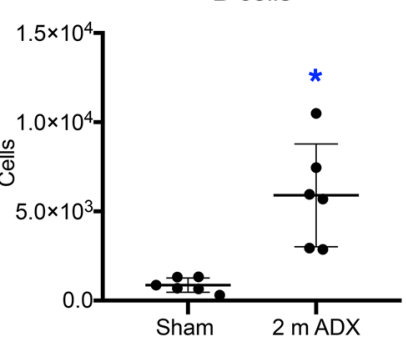

Macrophages

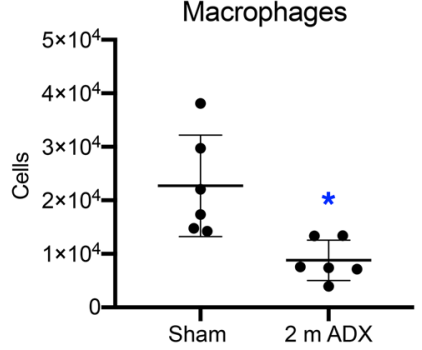

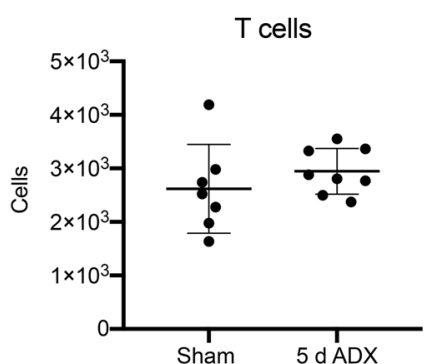
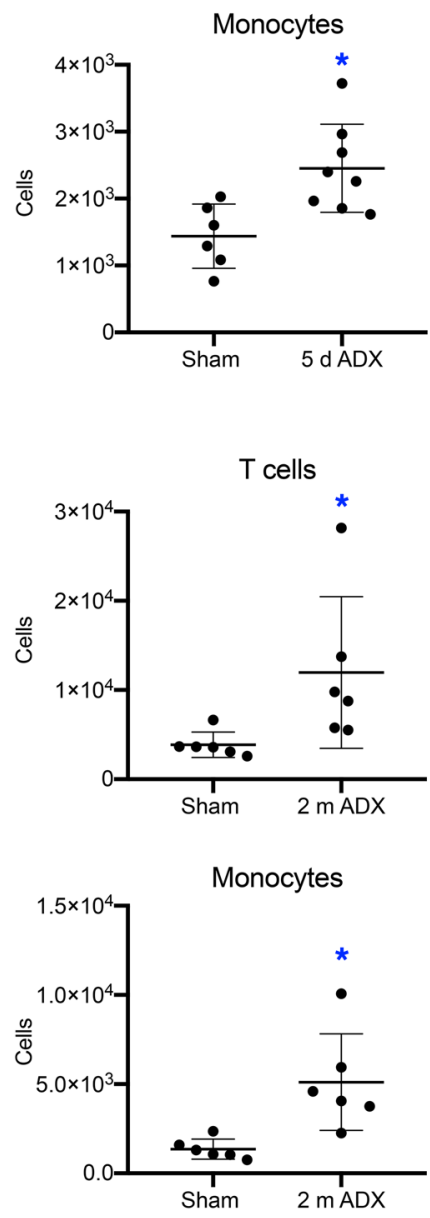

Figure 4. Adrenalectomy triggers chronic stomach inflammation.

Flow cytometric analysis of stomach leukocytes isolated from the gastric corpus lesser curvature 5 days $(\mathbf{A})$ or 2 months (B) after sham surgery or adrenalectomy. $P$ values were determined by unpaired 2-tailed $t$ test; $n=6$ mice/ group; ${ }^{*} P \leq 0.01,{ }^{* *} P \leq 0.001$. replacement prevented adrenalectomy-induced parietal cell and chief cell loss, SPEM development, cellular proliferation (Figure 2, $\mathrm{A}-\mathrm{C}$ and E), $\mathrm{CD}^{4} 5^{+}$leukocyte infiltration (Figure 2D), and induction of the SPEM-associated mRNAs Wfdc2, Olfm4, and $C f t r$ (Figure 2F). To determine if exogenous corticosterone could reverse the gastric pathology, mice were aged 1 month after adrenalectomy to develop SPEM then administered corticosterone in their drinking water for 1 week, 2 weeks, or 1 month (Supplemental Figure 4B). SPEM and oxyntic atrophy were fully developed 1 month after adrenalectomy (Supplemental Figure $4 \mathrm{C})$. Parietal cells recovered quickly in response to corticosterone replacement and were significantly increased from 16 cells per $\times 20$ field 1 month after adrenalectomy to 156 cells, 243 cells, and 302 cells per $\times 20$ field following 1 week, 2 weeks, and 1 month of corticosterone treatment, respectively (Supplemental Figure 4, C and E). The chief cell population followed a similar pattern and increased from only 1 cell per $\times 20$ field 1 month after adrenalectomy to 29 cells, 69 cells, and 140 cells per $\times 20$ field after 1 week, 2 weeks, and 1 month of corticosterone treatment, respectively. Leukocyte infiltration was resolved after 1 month of corticosterone treatment (Supplemental Figure 4D). These results suggest that endogenous glucocorticoids are necessary to maintain gastric homeostasis and that treatment with exogenous glucocorticoids can resolve SPEM in mice.

Endogenous glucocorticoids are required to suppress the transcription of proinflammatory genes within the stomach. Inflammation is a key driver of SPEM and gastric cancer development (30). Glucocorticoids are well known for their ability to suppress the transcription of proinflammatory genes. We observed chronic gastric inflammation following removal of endogenous glucocorticoids. However, the factors that initiate gastric inflammation remain unclear. We performed RNA sequencing using RNA isolated from the gastric corpus lesser curvature 3 days after sham surgery or adrenalectomy to investigate the mechanisms underlying adrenalectomy-induced gastric 


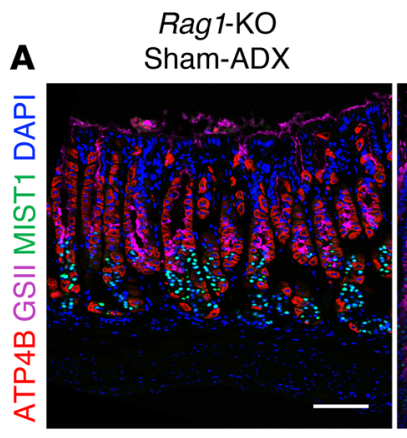

B

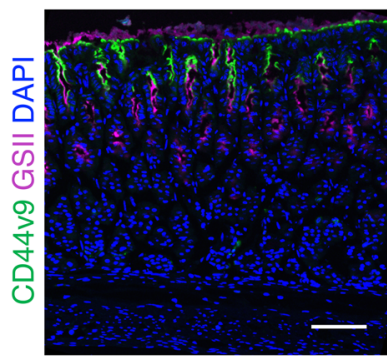

C

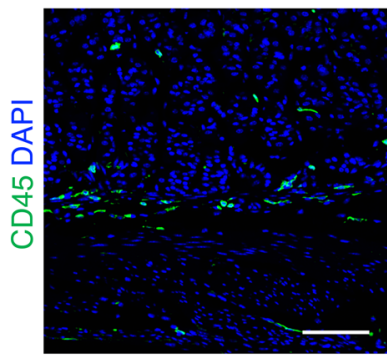

Rag1-KO
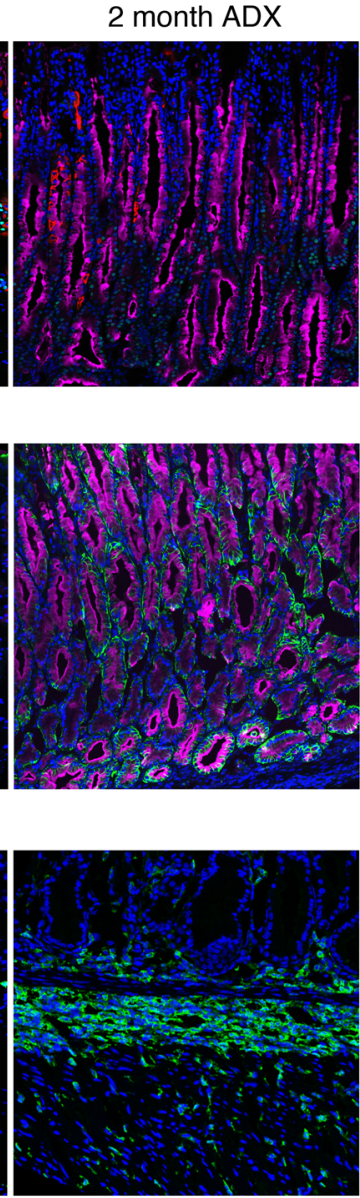
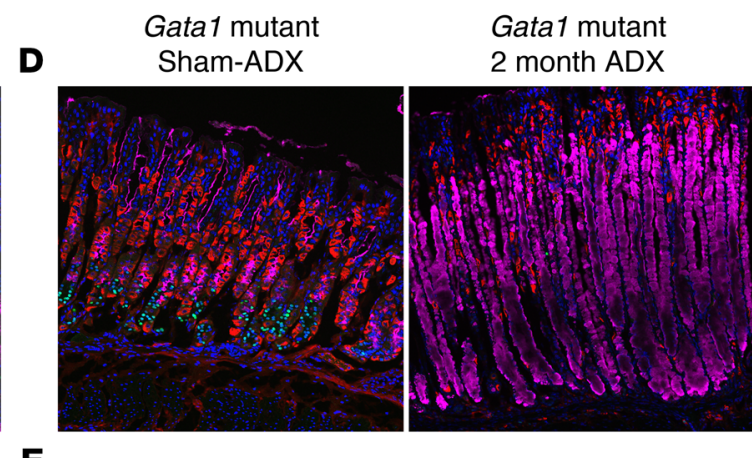

E
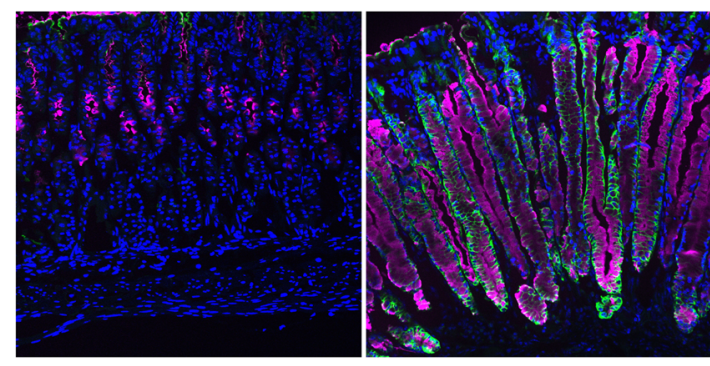

$\mathbf{F}$
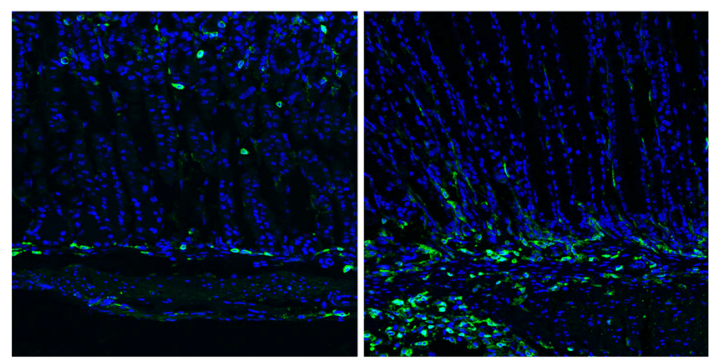

Figure 5. B cells, T cells, and eosinophils are dispensable for adrenalectomy-induced SPEM. Stomach sections from the gastric corpus lesser curvature of Rag1-KO mice (A-C) or Gata1 mutant mice (D-F) euthanized 2 months after sham surgery or adrenalectomy. Sections were probed for (A and D) ATP4B (parietal cells, red), GSII lectin (mucous neck cells, pink), and MIST1 (chief cells, green), (B and E) the SPEM marker CD44v9 (green) and GSII, or (C and F) CD45 (leukocytes, green). Nuclei were labeled with DAPI (blue). Scale bars: $100 \mu \mathrm{m} ; n=6$ mice/group.

inflammation and SPEM development. Importantly, histological examination revealed that the gastric corpus lesser curvature was normal 3 days after surgery and did not exhibit increased stomach leukocytes (Supplemental Figure 5). Thus, the induction of inflammatory genes precedes gastric leukocyte infiltration. RNA sequencing revealed that 1914 genes were significantly changed 3 days after adrenalectomy compared with sham controls (Supplemental Figure 6). To define the pathways that are altered in the stomach 3 days after adrenalectomy, the significant gene list was analyzed by Ingenuity Pathway Analysis (IPA), which revealed that the top 6 enriched canonical pathways all involved immune system function (Figure $3 \mathrm{~A})$. In addition, based on the changes in gene expression, IPA analysis predicted robust activation of leukocyte chemotaxis (Figure 3B). Gene set enrichment analysis (GSEA) further demonstrated significant activation of proinflammatory pathways, including the adaptive immune system, innate immune system, cytokine signaling pathways, and chemokine signaling pathways (Figure 3C). These data indicate that disruption of glucocorticoid signaling results in the rapid activation of proinflammatory gene networks within the stomach, thus suggesting that increased chemokine production by the stomach promotes the infiltration of circulating leukocytes. These data suggest that endogenous glucocorticoids play a critical role in suppressing spontaneous gastric inflammation.

Adrenalectomy triggers massive inflammation of the gastric corpus. Given that prominent inflammation occurs after adrenalectomy, we sought to characterize the inflammatory infiltrate to identify pathogenic leukocyte populations. Leukocytes were isolated from the lesser curvature of the gastric corpus from mice euthanized 5 days or 2 months after sham surgery or adrenalectomy (Supplemental Figure 7A). Adrenalectomy induced acute gastric inflammation and there was a significant 1.5-fold increase in total leukocytes 5 days after adrenalectomy (Figure 4A). The inflammatory infiltrate was principally composed of B cells, eosinophils, and monocytes (Figure 4A; see Supplemental Figure 7B for gating strategy). Analysis of the chronic gastric inflammation 2 months after adrenalectomy revealed a significant 4.2-fold increase in total leukocytes and a significant increase in B cells, T cells, eosinophils, and monocytes (Figure 4B). Interestingly, while the total number of monocytes was higher at both time points, macrophages were significantly decreased 2 months after adrenalectomy. At neither time point did we observe a significant change in the number of neutrophils or dendritic cells (Supplemental Figure 7C). 
A

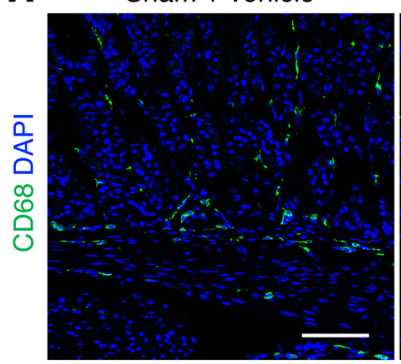

B

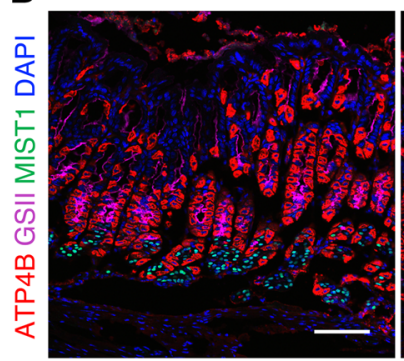

C

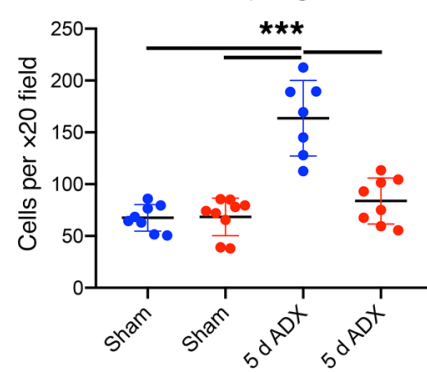

Sham + clodronate
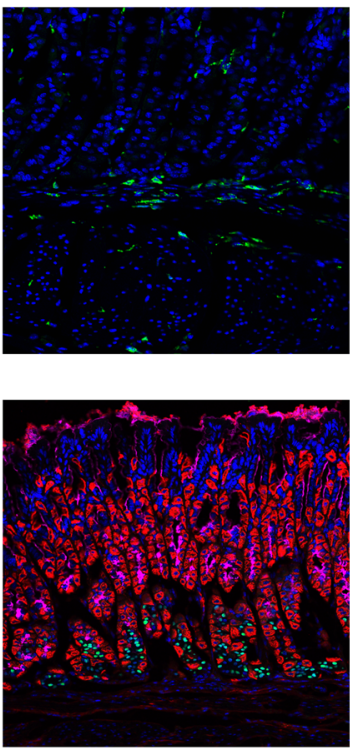

Parietal cells

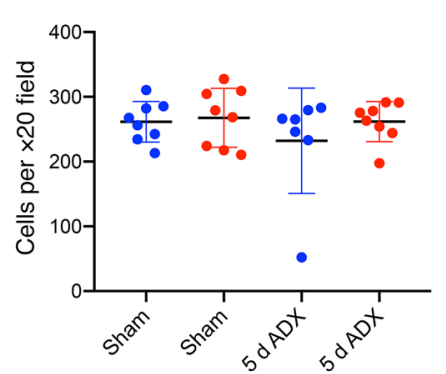

5 day $A D X+$ vehicle
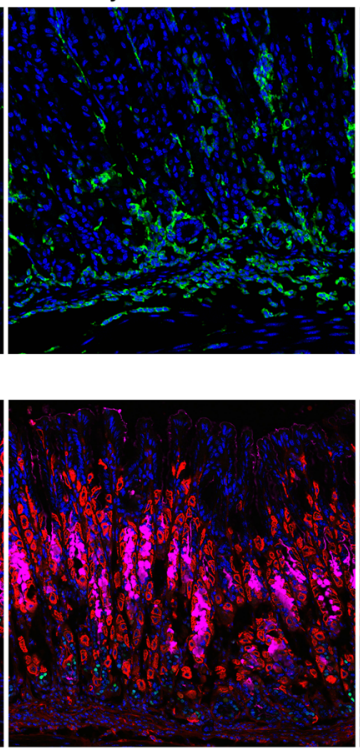

5 day $\mathrm{ADX}+$ clodronate
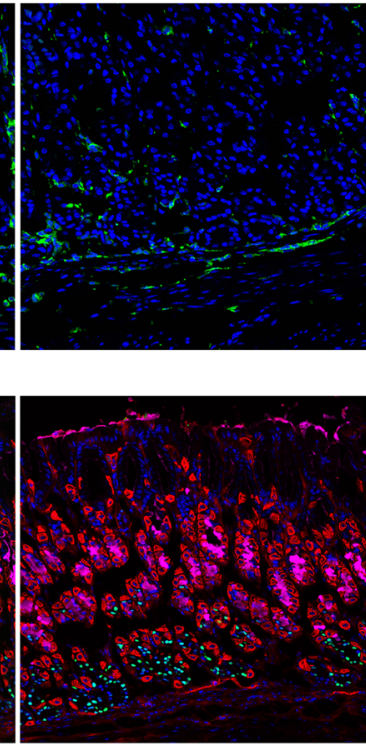

Chief cells

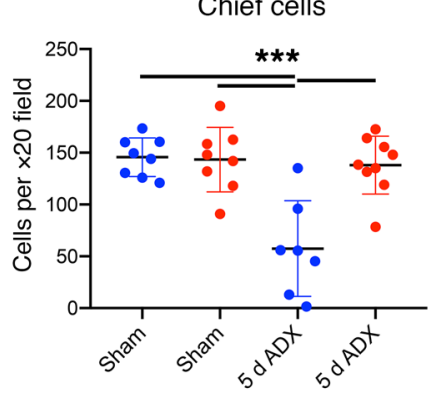

- Vehicle

- Clodronate

Figure 6. Monocyte/macrophage depletion inhibits adrenalectomy-induced SPEM development. Immunostaining of stomach sections from the gastric corpus lesser curvature of mice euthanized 5 days after sham surgery or adrenalectomy, treated with empty liposomes (vehicle) or clodronate. Sections were probed for (A) CD68 (macrophages, green) or (B) ATP4B (parietal cells, red), CSII lectin (mucous neck cells, pink), or MIST1 (chief cells, green). Nuclei were labeled with DAPI (blue). Scale bars: $100 \mu \mathrm{m}$. (C) Quantitation of the number of CD68+ macrophages, parietal cells, or chief cells observed per $\times 20$ field within the lesser curvature ( $n \geq 7$ mice/group). Data are mean \pm SD; $P$ values were determined by 1-way ANOVA with post hoc Tukey's $t$ test. ${ }^{* * *} P \leq 0.0001$.

$B$ cells, $T$ cells, and eosinophils are dispensable for initiating adrenalectomy-induced SPEM. Previous studies have found that T cells contribute to parietal cell death in Helicobacter-infected mice (4). Our RNAseq results indicated activation of $\mathrm{T}$ helper cell pathways (Figure 3A), and we observed a significant increase in $\mathrm{B}$ and $\mathrm{T}$ cells following adrenalectomy (Figure 4). Therefore, we adrenalectomized Rag1-KO mice, which lack mature B and T cells, to evaluate the contribution of the adaptive immune system in driving adrenalectomy-induced oxyntic atrophy and SPEM development (31). Adrenalectomized Rag1-KO mice exhibited oxyntic atrophy and loss of mature chief cells (Figure 5A) as well as induction of the SPEM marker CD44v9 (Figure 5B) 2 months after adrenalectomy. Furthermore, Rag1-KO mice exhibited a massive increase of $\mathrm{CD} 45^{+}$leukocytes (Figure 5C) as well as eosinophils and monocytes/macrophages (Supplemental Figure 8, A and B). These findings indicate that B and $\mathrm{T}$ cells are dispensable for adrenalectomy-induced oxyntic atrophy, SPEM development, and gastric inflammation.

Eosinophils are cytotoxic granulocytes associated with allergic reaction and antiparasitic immune responses within the intestines, and are a common feature of chronic gastric inflammation and SPEM $(32,33)$. Adrenalectomy robustly induced eosinophil infiltration into the stomach (Figure 1D and Figure 4). Therefore, we adrenalectomized homozygous Gata1 mutant mice ( $\triangle \mathrm{dblGA}$ $\mathrm{TA}$ ), which have a specific deletion of enhancer elements in the Gata1 promotor that disrupts eosinophil development (34), to determine if eosinophils were required for SPEM development. Two months after adrenalectomy, dblGATA mice exhibited oxyntic atrophy, loss of mature chief cells (Figure 5D), and induction of CD $44 \mathrm{v} 9$ expression which was not detectable in sham controls (Figure 5E). While the number of $\mathrm{CD} 45^{+}$leukocytes was markedly reduced (Figure 5F), this was attributed to the absence of eosinophils (Supplemental Figure 8C). However, the number of $\mathrm{CD} 68^{+}$cells was grossly similar to adrenalectomized WT mice (Supplemental Figure 8D). These data suggest that eosinophils are dispensable for adrenalectomy-induced oxyntic atrophy and SPEM development.

Monocyte/macrophages are critical mediators of SPEM development. Macrophages are the most abundant resident leukocyte in the stomach, and monocyte infiltration sharply increases after adrenalectomy (Figure 4). Macrophages have been previously shown to 
A
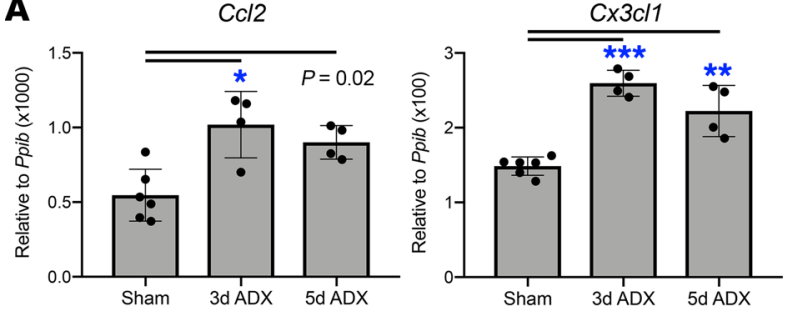

B

Col2
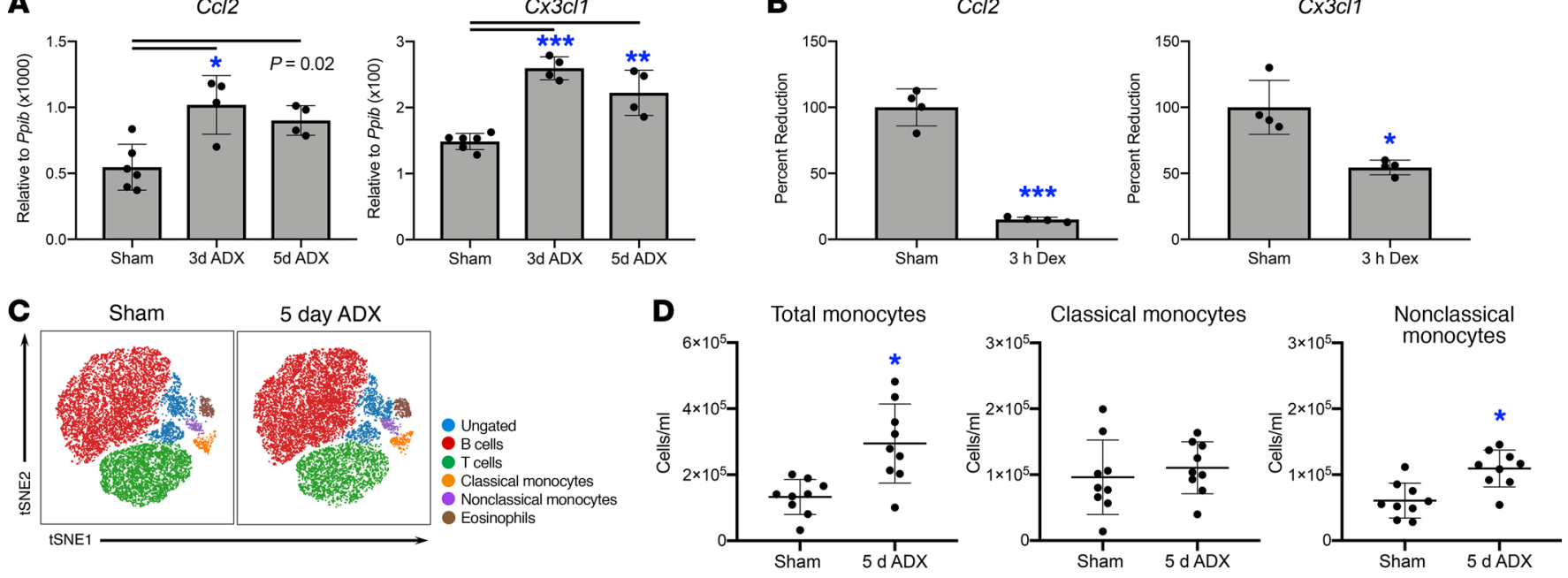

E

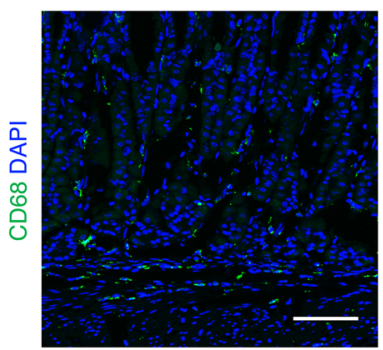

5 day ADX Ccr2-KO
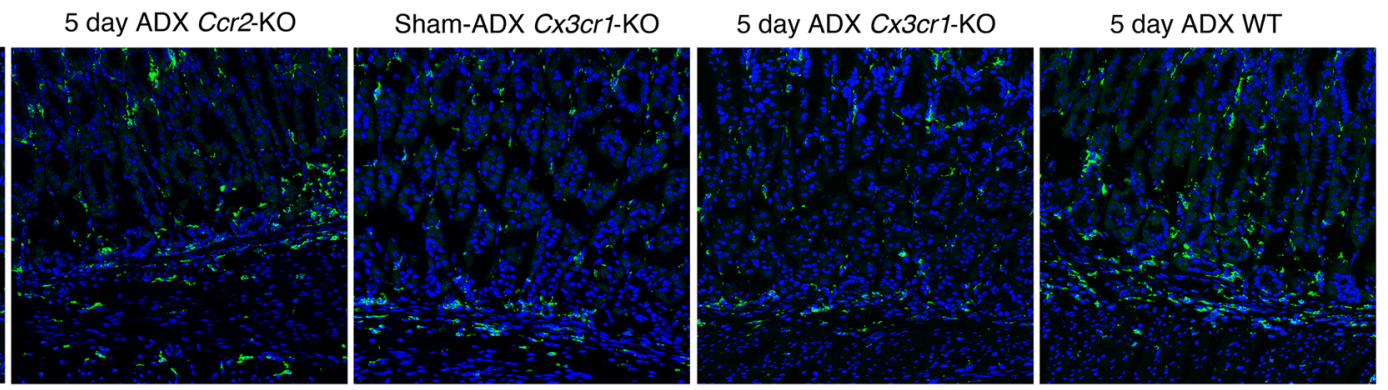

$\mathbf{F}$
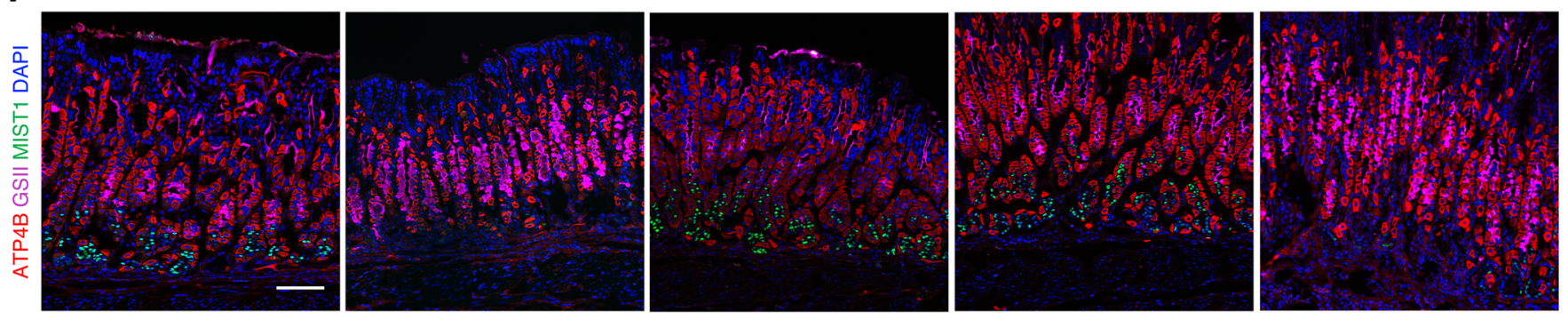

G
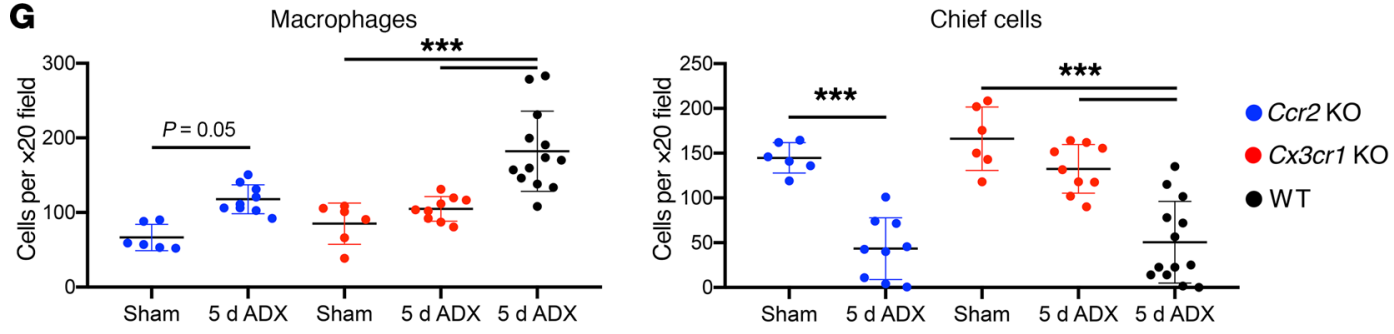

Figure 7. Infiltrating monocytes drive the development of gastric metaplasia. Quantitative RT-PCR of $C c / 2$ and $C x 3 c / 1$ using RNA isolated from the gastric corpus lesser curvature from mice euthanized (A) 3 or 5 days after sham surgery or adrenalectomy, or (B) from adrenalectomized mice euthanized 3 hours after treatment with $1 \mathrm{mg} / \mathrm{kg}$ dexamethasone ( $n \geq 4$ mice/group). (C) Representative tSNE analysis of circulating leukocytes by flow cytometry. (D) Quantitation of circulating monocytes ( $n \geq 9$ mice/group). (E-F) Immunostaining of stomach sections from Ccr2-KO, Cx3cr1-KO, or WT mice euthanized 5 days after sham surgery or adrenalectomy. Sections were probed for (E) CD68 (macrophages, green) or (F) ATP4B (parietal cells, red), GSII lectin (mucous neck cells, pink), and MIST1 (chief cells, green). Nuclei were labeled with DAPI (blue). Scale bars: $100 \mu \mathrm{m}$. (G) Quantitation of the number of CD68 ${ }^{+}$monocytes/ macrophages or mature chief cells observed per $\times 20$ field within the lesser curvature ( $n \geq 6$ mice/group). All data are mean \pm SD; $P$ values were determined by 1-way ANOVA with post hoc Tukey's $t$ test (A-B and $\mathbf{G}$ ) or by unpaired 2-tailed $t$ test (D). ${ }^{*} P \leq 0.01,{ }^{* *} P \leq 0.001,{ }^{* * *} P \leq 0.0001$.

contribute to SPEM development $(35,36)$. Therefore, we depleted the monocyte lineage with clodronate liposomes to determine their role in acute SPEM development 5 days after adrenalectomy. Mice received daily clodronate injections beginning 24 hours before sur- gery and were euthanized 5 days after surgery (Supplemental Figure 9A). Clodronate treatment of sham mice did not deplete tissue-resident macrophages (Figure 6, A and C) which is consistent with a previous report that clodronate treatment does not deplete stomach 

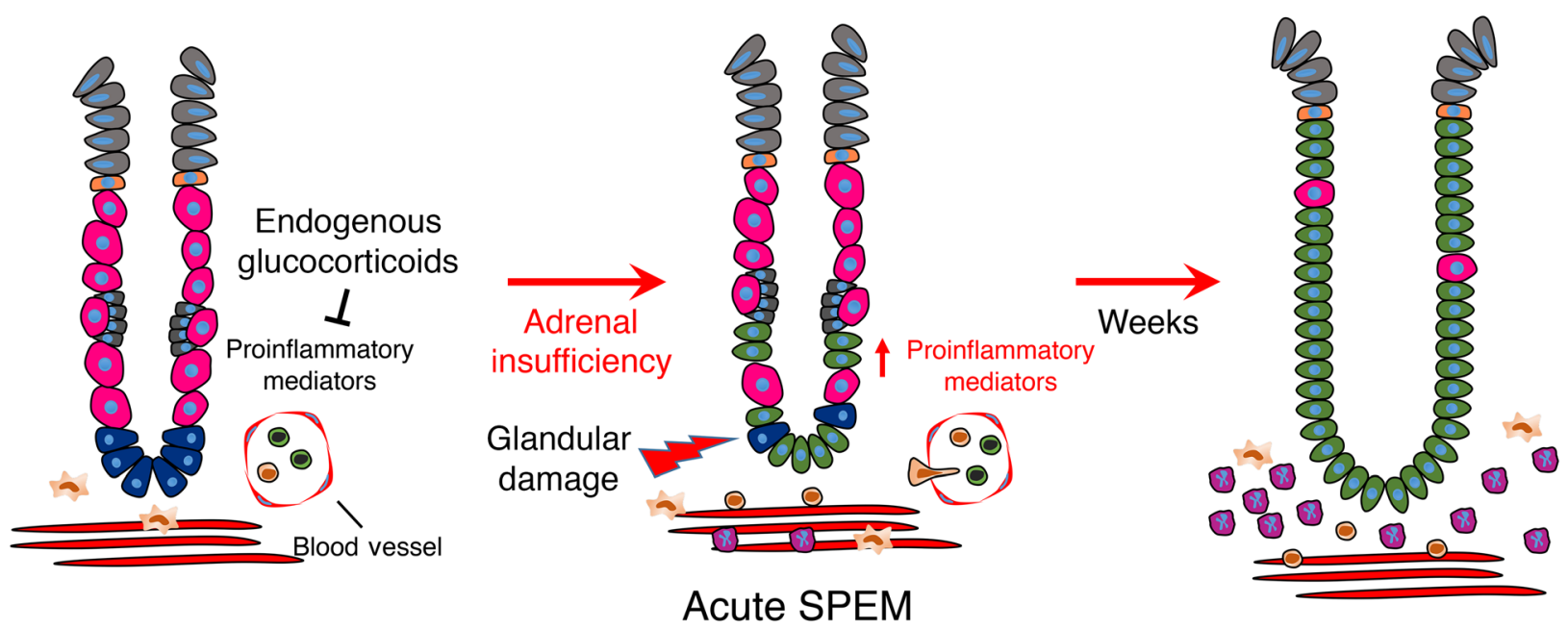

\begin{tabular}{|lll|}
\hline Pit (foveolar) cell & 0 CX3CR1 ${ }^{+}$monocyte \\
Gland stem cell & 0 CCR2 ${ }^{+}$monocyte \\
Parietal cell & $\sim$ Macrophage \\
Mucous neck cell & 0 Eosinophil \\
\hline Chief cell & 0 SPEM cell \\
\hline
\end{tabular}

\section{Chronic SPEM}

Figure 8. Endogenous glucocorticoids are required to maintain gastric homeostasis. Under steady-state conditions, endogenous glucocorticoids suppress the expression of proinflammatory mediators in the gastric corpus. Loss of endogenous glucocorticoids results in the production of proinflammatory mediators, leading to infiltration of circulating monocytes and eosinophils. Infiltrating CX3CR1 $1^{+}$monocytes exhibit pathogenic activation due to disrupted glucocorticoid signaling, and damage the gastric epithelium, leading to SPEM development. Over the course of weeks, parietal cells are lost and the gastric gland becomes predominately filled with metaplastic cells which over time may create a favorable environment for the evolution of gastric adenocarcinoma.

resident macrophages (36). Five days after adrenalectomy, vehicle-treated mice exhibited a 2.4-fold increase in stomach $\mathrm{CD} 68^{+}$ monocytes/macrophages and a $61 \%$ loss of mature chief cells (Figure 6). In contrast, clodronate treatment blocked the expansion of $\mathrm{CD}^{+} 8^{+}$cells in the stomach and prevented loss of mature chief cells. Vehicle-treated adrenalectomized mice exhibited increased GSII staining that reached the bottom of the gastric glands (Figure 6B), and robust induction of CD44v9 (Supplemental Figure 9B) indicating SPEM development. However, clodronate treatment prevented adrenalectomy-induced SPEM. There was no change in proliferation 5 days after adrenalectomy or in adrenalectomized mice treated with clodronate (Supplemental Figure 9C). Parietal cell loss has long been thought to be an obligate precursor of SPEM development (30). However, a recent study reported that SPEM development was avoided when parietal cells died by apoptosis following treatment with diphtheria toxin (37), thus challenging the notion that parietal cell depletion is sufficient to drive SPEM. We found that although there was robust SPEM development 5 days after adrenalectomy (Supplemental Figure 9B), there was not a corresponding reduction in parietal cells (Figure 6, B and C). Together, these results suggest that infiltrating monocytes are required for loss of mature chief cells and SPEM development after adrenalectomy, and that parietal cell loss is not required to induce SPEM development.

CX $3 C R 1^{+}$monocytes are critical mediators of SPEM development. Because clodronate treatment did not deplete stomach resident macrophages, we hypothesized that infiltration by circulating monocytes was required for SPEM development. Therefore, we evaluated the expression of the chemokines $C c l 2$ and $C x 3 c l 1$ within the gastric corpus, which are critical for recruiting monocytes from circulation. Quantitative RT-PCR showed that both chemokines were significantly upregulated 3 and 5 days after adrenalectomy (Figure 7A). Expression of $\mathrm{Ccl} 2$ and $\mathrm{C} \times 3 \mathrm{cl} 1$ are directly suppressed by the glucocorticoid receptor $(38-40)$. We investigated whether glucocorticoids suppress the expression of these chemokines in the stomach by treating mice with $1 \mathrm{mg} / \mathrm{kg}$ dexamethasone 3 days after adrenalectomy. Three hours after dexamethasone treatment, there was an $85 \%$ and $46 \%$ suppression of $C c l 2$ and Cx3cl1 mRNA in the stomach, respectively (Figure 7B). Circulating murine monocytes are subdivided into 2 groups based on their differential expression of the chemokine receptors CCR2 and CX3CR1 and the surface marker LY6C (41, 42). Classical monocytes are defined as LY6C ${ }^{\text {positive }}, \mathrm{CCR} 2^{\text {hi }}$, and CX3CR $1^{\text {lo }}$ whereas nonclassical monocytes are $\mathrm{LY} \mathrm{C}^{\text {negative }}, \mathrm{CCR} 2^{\text {lo }}$, and CX3CR $1^{\text {hi }}$ $(43,44)$. We used flow cytometry to assess changes in circulating monocytes 5 days after adrenalectomy (see Supplemental Figure $10 \mathrm{~A}$ for gating strategy). We found that there was a significant increase in the total number of monocytes $\left(\mathrm{SSC}^{\mathrm{lo}} \mathrm{F} 4 / 80^{+} \mathrm{CD} 11 \mathrm{~b}^{+}\right)$ 5 days after adrenalectomy (Figure 7, $C$ and $D$ ). In addition, there was a significant increase in $\mathrm{B}_{2} 2 \mathrm{O}^{+} \mathrm{B}$ cells, whereas the number of $\mathrm{T}$ cells, natural killer cells, eosinophils, and neutrophils did not significantly change (Supplemental Figure 10, B and C). Five days after adrenalectomy, the number of circulating classical monocytes was unchanged (Figure 7, C and D) but the number of nonclassical monocytes was significantly increased. 
Adrenalectomy upregulated the expression of monocyte chemokines by the stomach and increased the number of circulating monocytes. Consequentially, we next adrenalectomized Ccr2$\mathrm{KO}$ mice and $\mathrm{Cx} 3 \mathrm{cr} 1-\mathrm{KO}$ mice to test if recruitment of a subset of circulating monocytes is required for SPEM development. Both Ccr2-KO and Cx3cr1-KO are sham controls had a normal number of $\mathrm{CD} 68^{+}$cells in the gastric corpus indicating that the stomach resident macrophage population is maintained in these mouse models (Figure 7, E and G). Five days after adrenalectomy, Ccr2KO mice exhibited blunted expansion of stomach $\mathrm{CD}^{+} 8^{+}$monocytes/macrophages compared with adrenalectomized WT mice (Figure 7, E and G). Despite this blunted inflammatory response, Ccr2-KO mice exhibited a significant loss of mature chief cells 5 days after adrenalectomy (Figure 7, F and G) and equivalent SPEM development compared with WT mice (Supplemental Figure 11). In contrast, there was no change in $\mathrm{CD}^{+} 8^{+}$cells 5 days after adrenalectomy in the stomachs of $\mathrm{C} x 3 \mathrm{cr} 1-\mathrm{KO}$ mice (Figure 7, $\mathrm{E}$ and $\mathrm{G}$ ). Furthermore, adrenalectomized $C x 3 c r 1-K O$ mice did not exhibit a significant loss of mature chief cells (Figure 7, F and G) and did not develop SPEM (Supplemental Figure 11). Interestingly, SPEM development was not detected in either adrenalectomized mice treated with clodronate (Supplemental Figure 9B) nor in adrenalectomized Cx3cr1-KO mice (Supplemental Figure 11) despite normal numbers of tissue-resident macrophages. Thus, tissue-resident macrophages may be insufficient to drive SPEM development. These data suggest that circulating monocytes are recruited to the stomach following adrenalectomy and that infiltrating monocytes/ macrophages, but not tissue-resident macrophages, are required for SPEM development. In addition, these findings suggest that the CX3CR1 $1^{+}$monocyte subpopulation, rather than CCR2 ${ }^{+}$monocytes, promote gastric metaplasia development.

\section{Discussion}

In this study, we used a combination of systemic adrenal insufficiency and genetically altered mouse models to test the requirement for glucocorticoid signaling in the stomach. We found that glucocorticoids were required to maintain gastric homeostasis and that adrenalectomy resulted in pathogenic gastric inflammation, leading to potentially preneoplastic SPEM development and oxyntic atrophy. Adrenalectomy led to rapid upregulation of proinflammatory gene networks within the gastric corpus 3 days after surgery, followed by massive expansion of the stomach leukocyte population. These results suggest that the stomach is primed for immune responsiveness and that a critical role of endogenous glucocorticoids is to suppress the production of proinflammatory cytokines. Among these upregulated genes were the monocyte chemokines $C c l 2$ and $C x 3 c l 1$, and we demonstrated that glucocorticoids suppress their expression in the gastric corpus. Monocyte and macrophages are a diverse category of immune cells that have a wide variety of functions ranging from maintaining tissue homeostasis to protecting from infection. Dysregulation of these cells is linked to the development and progression of a wide variety of cancers $(45,46)$. Using the Crr2-KO and $\mathrm{Cx} 3 \mathrm{cr} 1$-KO mouse models, we demonstrated that only the CX3CR $1^{+}$monocyte subpopulation was required to drive SPEM development. In contrast, CCR2 ${ }^{+}$monocytes were not required for SPEM development. Glucocorticoids have an established role in suppressing pathogenic activation of monocytes and macrophages, and deletion of the GR in the monocyte lineage leads to their pathogenic activation after challenge with lipopolysaccharide and is fatal in mice (47). Thus, in addition to regulating gastric monocyte recruitment, glucocorticoids also regulate their activity to prevent pathogenic monocyte/macrophage activation. Taken together, our results support that glucocorticoid signaling plays a critical role in suppressing pathogenic immune activation in the stomach and suggest that disruption of glucocorticoid signaling may promote the development of gastric neoplasia (a model can be found in Figure 8).

Chronic inflammation and metaplasia form the requisite environment for the development of gastric cancer. $H$. pylori infection is the most common risk factor for gastric cancer in humans and is associated with nearly $90 \%$ of cancer cases (2). Despite $H$. pylori infecting approximately $50 \%$ of the world population, only $1 \%-3 \%$ of infected individuals will progress to develop gastric cancer $(48,49)$. It has long been suspected that a pathogenic switch in the inflammatory phenotype favors gastric cancer development in a subset of infected individuals. Factors that promote more aggressive inflammation are linked to poorer patient prognosis. For instance, $\mathrm{CagA}^{+}$strains of $H$. pylori elicit more robust inflammation and are associated with a higher incidence of cancer $(50,51)$. Furthermore, polymorphisms in genes encoding the inflammatory mediators IL1B or NFKB1 dramatically increase the risk of gastric cancer in $H$. pylori-infected individuals $(9,52,53)$, and can even promote cancer development in the absence of infection (6-8). Here we demonstrate that signaling by endogenous glucocorticoids is required to suppress pathogenic gastric inflammation metaplasia. Glucocorticoids are most well-known for their ability to limit the intensity and duration of an inflammatory response, and systemic adrenal insufficiency is tied to the development of chronic inflammatory and autoimmune diseases. Clinical studies have reported that adrenal-insufficient patients often exhibit autoimmune and inflammatory gastritis $(14,15)$. However, adrenal insufficiency such as Addison's disease is not associated with an increased incidence of gastric cancer, presumably because these patients receive glucocorticoid replacement therapy. In this study, we demonstrated that glucocorticoid replacement is sufficient to suppress gastric inflammation and metaplasia in adrenal-insufficient mice. It is important to note that chronic inflammation can override local glucocorticoid activity, leading to tissue-specific glucocorticoid resistance and uncontrolled pathogenic inflammation despite normal levels of circulating glucocorticoids (12, 54). Thus, chronic inflammation caused by long-term $H$. pylori infection may disrupt glucocorticoid signaling within the stomach, causing pathogenic inflammation and promoting cancer development. Indeed, analysis of human stomach cancer biopsies supports this concept, reporting that expression of the GR is reduced in stomach cancer $(16,17)$.

Gastric adenocarcinoma is the most prevalent type of gastric cancer, and follows a well-established model of progression from atrophic gastritis, intestinal metaplasia, dysplasia, to adenocarcinoma $(49,55)$. Current evidence suggests that SPEM development precedes intestinal metaplasia and that SPEM transitions to intestinal metaplasia or progresses directly to gastric adeno- 
carcinoma (3). Until recently, parietal cell death was considered an obligate precursor of SPEM development (1). Drug models of acute SPEM such as treatment with a high dose of tamoxifen or the proton ionophore L635 induce SPEM by killing parietal cells $(19,56)$. However, a recent report found that SPEM did not develop after parietal cell apoptosis using a Cre-inducible diphtheria toxin receptor model. Thus, this report challenges the notion that parietal cell loss is sufficient to drive metaplasia development (37). In our model, acute SPEM development occurred 5 days after adrenalectomy before there was any change in the parietal cell population, but parietal cell loss did eventually occur, as there was an $82 \%$ reduction of parietal cells 2 months after adrenalectomy. A potential explanation for this finding is that SPEM development may represent an adaptive response to damage within the gastric corpus rather than solely a response to parietal cell loss. Supporting this concept, it has been shown that acute SPEM promotes healing and resolution of acetic acid-induced ulcers $(20,23)$. To our knowledge, this is the first study to show SPEM development before parietal cell loss. Thus, adrenalectomy as an experimental model of SPEM has a unique advantage over existing models, which require chemical ablation of parietal cells to induce SPEM development.

Macrophages have been linked to the development of preneoplastic metaplasia in the stomach $(4,35,36)$. Our findings agree with these reports and suggest that the monocyte/macrophage lineage is required to initiate SPEM development. Peterson et al. recently reported that $\mathrm{M} 2 \mathrm{a}$ polarized macrophages were required for SPEM development after chemical parietal cell depletion (35). In a separate study, the same research group found that tissue-resident macrophages were insufficient to drive SPEM development (36), raising the possibility that infiltrating monocyte-derived macrophages may be required for SPEM development. Here we showed that adrenalectomy led to an increase in circulating nonclassical monocytes and that deletion of the fractalkine receptor Cx3cr1 prevented gastric monocyte infiltration and SPEM development. In contrast, adrenalectomized Crr2-KO mice exhibited reduced monocyte infiltration but they were not protected from SPEM development. Some reports have suggested that nonclassical monocytes are primed to differentiate toward M2 macrophages, supporting the notion that nonclassical monocytes may promote metaplasia in the stomach $(57,58)$. Small molecule inhibitors have been effectively used to target both CX3CR1 and its ligand CX3CL1, thereby preventing macrophage-induced inflammatory diseases (59-61). Thus, CX3CR1 may represent a novel and effective target to treat gastric inflammation and SPEM in humans.

In conclusion, we have found that endogenous glucocorticoids are required to suppress gastric inflammation and SPEM development. We also demonstrated that acute SPEM development precedes oxyntic atrophy and that SPEM development depends on gastric infiltration by $\mathrm{CX} 3 \mathrm{CR} 1^{+}$monocytes. These results provide novel insights into the normal physiology of the stomach as well as the mechanisms that regulate pathogenic gastric inflammation and metaplasia. Thus, endogenous glucocorticoids may represent a critical barrier to proneoplastic gastric inflammation and disrupting glucocorticoid signaling may set the stage for gastric cancer development.

\section{Methods}

Animal care. Female WT C57BL/6J mice (catalog 000664), Rag1-KO mice (catalog 002216), Gata1 mutant mice (catalog 005653), Cx3cr1KO mice (catalog 005582), and Ccl2-KO mice (catalog 004999) were purchased from the Jackson Laboratories. All mice were maintained on a C57BL/6J genetic background with the exception of Gata1 mutant mice, which were on a congenic BALB/c genetic background. Mice were administered standard chow and water ab libitum and maintained in a temperature- and humidity-controlled room with standard 12-hour light/dark cycles. Sham-adrenalectomy and adrenalectomy surgeries were performed by the Jackson Laboratories or by the NIEHS comparative medicine branch when mice were 8 weeks of age. Following adrenalectomy, mice were maintained on $0.85 \%$ saline drinking water to maintain ionic homeostasis.

Corticosterone measurement. Adrenalectomy was confirmed by ELISA. Serum was collected from sham-adrenalectomy and adrenalectomized mice to determine the levels of circulating glucocorticoids. The corticosterone concentration was determined by a commercially available ELISA kit (Arbor Assays), according to the manufacturer's protocol.

Tissue preparation. Mice were euthanized by cervical dislocation without anesthesia 3 days, 5 days, or 2 months after surgery. Adrenalectomy was confirmed by corticosterone ELISA and by checking for the absence of the adrenal glands upon euthanasia. Stomachs were removed and opened along the greater curvature and washed in 2 changes of ice-cold phosphate-buffered saline to remove gastric contents. Stomachs used for histology were pinned to cork boards and fixed overnight in $4 \%$ paraformaldehyde at $4^{\circ} \mathrm{C}$. Following fixation, stomachs used for cryosectioning were cryopreserved in 30\% sucrose and then embedded in optimal cutting temperature (OCT) media. Stomachs used for paraffin sectioning were submitted to the NIEHS histology core for routine processing, embedding, sectioning, and H\&E staining. For RNA isolation, the region of interest was collected with a 4-mm biopsy punch and immediately snap-frozen in liquid nitrogen. RNA was extracted in TRIzol (Thermo Fisher Scientific) and precipitated from the aqueous phase using 1.5 volumes of $100 \%$ ethanol. The mixture was transferred to a RNeasy column (Qiagen), and the remaining steps were followed according to the RNAeasy kit manufacturer's recommendations. RNA was treated with RNase free DNase I (Qiagen) as part of the isolation procedure.

Steroid and drug treatments. Adrenalectomized mice were administered corticosterone through their drinking water as previously described (62). Briefly, corticosterone (Steraloids) was dissolved in $100 \%$ ethanol, which was subsequently diluted $1: 200$ in $0.85 \%$ saline water. The final corticosterone concentration was $0.25 \mathrm{mg} / \mathrm{ml}$. This concentration has previously been shown to result in physiological levels of corticosterone in adrenalectomized mice (62). Control sham mice received normal drinking water while control adrenalectomized mice received $0.85 \%$ saline water. Hormone treatment was initiated either 1 week or 1 month after adrenalectomy, and mice were euthanized after 0 days, 1 week, 2 weeks, 1 month, or 2 months of continuous treatment.

Three days after adrenalectomy, mice were administered a single intraperitoneal injection of $1 \mathrm{mg} / \mathrm{kg}$ dexamethasone (Steraloids) in sterile PBS and euthanized 3 hours after treatment.

Sham and adrenalectomized mice were treated with clodronate-loaded liposomes via intraperitoneal injection to deplete monocytes and macrophages. Mice were injected with $10 \mathrm{mg} / \mathrm{kg}$ clodronate-loaded liposomes or equal volume of control liposomes (Encapsula NanoScienc- 
es). Injections were administered daily. The first dose was administered 1 day prior to surgery and continued for 4 days after surgery. Mice were euthanized 24 hours after the final treatment.

Histology. Immunostaining was performed using standard methods. Briefly, $5-\mu \mathrm{m}$ stomach cryosections were incubated with the primary antibodies listed in Supplemental Table 1 for 1 hour at room temperature or overnight at $4^{\circ} \mathrm{C}$. Primary antibody was omitted as a negative control. Following stringency washes, sections were incubated in secondary antibodies for 1 hour at room temperature. Fluorescence-conjugated phalloidin (1:500; Thermo Fisher Scientific) or fluorescence-conjugated Griffonia simplicifolia lectin (GSII; 1:500; Thermo Fisher Scientific) were added with secondary antibodies. Sections were mounted with Vectastain mounting media containing DAPI (Vector Laboratories). Images were obtained using a Zeiss 880 confocal laser-scanning microscope equipped with Airyscan (CarlZeiss) and running Zen Black imaging software (Carl-Zeiss). Paraffin sections were stained with H\&E using standard methods and were imaged by an Aperio AT2 Scanner (Leica Biosystems Inc.).

Image quantitation. Parietal cells, chief cells, and monocytes/ macrophages were quantitated manually using confocal micrographs captured using a $\times 20$ microscope objective and $1-\mu \mathrm{m}$ thick optical section. Cells were counted using the Image (NIH) count tool. Cells that stained positive with anti-ATP4B antibodies were identified as parietal cells, whereas cells that stained positive with anti-MIST1 antibodies and were GSII negative were identified as mature chief cells. Cells that stained positive with anti-CD68 antibodies were considered monocytes/macrophages. To be included in counts, cells were required to have a distinct DAPI-stained nucleus. Counts were reported as the number of cells observed per $\times 20$ field. Images were chosen that contain gastric glands cut longitudinally. Quantitation of $\mathrm{KI} 67^{+}$epithelial cells was performed using Imaris Cell $\times 64$ Imaging Software version 8.4.1 (Bitplane). $\mathrm{KI} 67^{+}$cells were identified using the following settings: $0.642-\mu \mathrm{m}$ filter width, cell threshold intensity of $50,5-\mu \mathrm{m}$ seed point diameter, 10.5 quality threshold, and 10 cell number of voxels. Epithelial cells were distinguished by coimmunostaining for the epithelial marker CTNNB1, and negative cells were removed from the analysis using a minimum mean intensity threshold of 25.

Flow cytometry. Flow cytometric analysis was performed using leukocytes isolated from the gastric corpus lesser curvature. Mice were euthanized by cervical dislocation and perfused through the left ventricle with $10 \mathrm{ml}$ PBS. The stomach was then removed and the lesser curvature adjacent to each side of the esophagus was removed with a 4-mm biopsy punch. Stomach tissue was washed 2 times in Hanks' balanced salt solution with $5 \mathrm{mM}$ HEPES, $5 \mathrm{mM}$ EDTA, and 5\% FBS for 20 minutes at $37^{\circ} \mathrm{C}$. Tissue was then digested in $1 \mathrm{mg} / \mathrm{ml}$ type $1 \mathrm{col}-$ lagenase (Worthington Biochemical) and $1.5 \mathrm{mg} / \mathrm{ml}$ DNaseI (Roche Molecular Systems) for 20 minutes at $37^{\circ} \mathrm{C}$. After tissue digestion, cells were enriched using an 18\% Optiprep gradient (Axis-Shield Diagnostics). Cells were stained with the fluorescent antibodies listed in Supplemental Table 1 for 20 minutes at $4^{\circ} \mathrm{C}$. Fc receptors were blocked with CD16/32 antibodies. Actinomycin D (7-AAD) was used to label dead cells. After staining, the cells were counted on a TC20 automated cell counted (Bio-Rad Laboratories). Blood was collected by submandibular vein puncture and the red blood cells were lysed with ACK buffer. Cells were counted on a hemocytometer and 100,000 cells were stained with the appropriate fluorescent antibodies as described above. Cells were analyzed by a LSRII Cytometer (BD Bioscience) and the data were analyzed by Cytobank software. Viable CD $45^{+}$cells were used for tSNE analysis.

Next-generation sequencing. RNA was isolated 3 days after sham surgery or adrenalectomy as described above. Each experimental group used 4 mice. Indexed samples were sequenced using the 75-bp paired-end protocol via the NextSeq500 (Illumina) per the manufacturer's protocol. Reads (34-76 million reads per sample) were aligned to the UCSC mm10 reference genome using STAR with default parameters. The quantification results from featureCounts were then analyzed with the Bioconductor package DESeq2, which fits a negative binomial distribution to estimate technical and biological variability. Comparisons were made between vehicleand dexamethasone-treated intact and adrenalectomized mice. A gene was considered differentially expressed if the $P$ value for differential expression was less than 0.01. Volcano plots were generated using the Partek Genomic Suite (version 6.6). Lists of significant genes were further analyzed using Ingenuity Pathway Analysis (content version 36601845, Ingenuity Systems). Enrichment or overlap was determined by IPA using Fisher's exact test $(P<0.05)$. GSEA analysis was performed using GSEA v2.2.2 software. Genes were preranked based on $P$ value and their fold change of gene expression. This application scores a sorted list of genes with respect to their enrichment of selected functional categories (Kyoto Encyclopedia of Genes and Genomes [KEGG], Biocarta, Reactome, and Gene Ontology [GO]). The significance of the enrichment score was assessed using 1000 permutations. Benjamini and Hochberg's false discovery rate (FDR) was calculated for multiple testing adjustments. A q value of less than 0.05 was considered significant. The resulting enriched pathways were visualized using the Cytoscape (v3.3.0) Enrichment Map plugin. The RNA-seq data are available in the Gene Expression Omnibus repository at the National Center for Biotechnology Information (https://www.ncbi.nlm.nih.gov/geo/) (accession number GSE107736).

Quantitative RT-PCR. To amplify mature mRNAs, primers spanning gene introns were used to avoid amplifying genomic DNA. Reverse transcription followed by qRT-PCR was performed in the same reaction using a Universal Probes One-Step PCR kit (Bio-Rad Laboratories) and the Taqman primers (Thermo Fisher Scientific) listed in Supplemental Table 2 following the manufacturers recommended settings on a CFX96 Real-Time PCR system (Bio-Rad Laboratories). All reactions were performed in duplicate using 50 ng total RNA. Messenger RNA levels were normalized to the reference gene Ppib, which remained unchanged in our experimental conditions, and data were presented using either the $\Delta \mathrm{Ct}$ or $\Delta \Delta$ Ct method.

Statistics. All error bars are \pm the standard deviation of the mean. Sample size for each experiment is indicated in the figure legends. Experiments were repeated a minimum of 2 times. Statistical analyses were performed using 1-way ANOVA with post hoc Tukey's $t$ test when comparing 3 or more groups or by unpaired $t$ test when comparing 2 groups. Statistical analysis was performed by Graphpad Prism 7 software. Statistical significance was set at $P \leq 0.01$. Specific $P$ values are listed in the figure legends.

Study approval. All animal studies were performed with approval from the Animal Care and Use Committee of the National Institute of Environmental Health Sciences (NIEHS). 


\section{Author contributions}

JTB was responsible for study design, performing experiments, data collection, data interpretation, and drafted the manuscript. SR and DWC performed experiments, interpreted data, and edited the manuscript. XX analyzed the RNA sequencing data. DNC performed data interpretation and edited the manuscript. JAC developed the experimental model, provided funding, and edited the manuscript.

\section{Acknowledgments}

The authors thank the NIEHS Comparative Medicine Branch, Histology Core Laboratory, Epigenomes Core Laboratory, and Fluores- cence Microscopy and Imaging Center for their assistance. We also thank Robert Oakley (NIEHS) for critical reading of this manuscript. This research was supported by the Intramural Research Program of the NIH/NIEHS 1ZIAES090057 (to JAC) and by a Postdoctoral Research Associate (PRAT) fellowship from the National Institute of General Medical Sciences 1Fi2GM123974 (to JTB).

Address correspondence to: John A. Cidlowski, Building 101 MD F3-07, 111 TW Alexander Drive, Research Triangle Park, North Carolina 27709, USA. Phone: 984.287.3545; Email: cidlows1@ niehs.nih.gov.
1. Goldenring JR, Nam KT, Wang TC, Mills JC, Wright NA. Spasmolytic polypeptide-expressing metaplasia and intestinal metaplasia: time for reevaluation of metaplasias and the origins of gastric cancer. Gastroenterology 2010;138(7):2207-2210, 2210.e1.

2. Plummer M, Franceschi S, Vignat J, Forman D, de Martel C. Global burden of gastric cancer attributable to Helicobacter pylori. Int J Cancer. 2015;136(2):487-490.

3. Yoshizawa N, et al. Emergence of spasmolytic polypeptide-expressing metaplasia in Mongolian gerbils infected with Helicobacter pylori. $\mathrm{Lab}$ Invest. 2007;87(12):1265-1276.

4. Roth KA, Kapadia SB, Martin SM, Lorenz RG. Cellular immune responses are essential for the development of Helicobacter felis-associated gastric pathology. JImmunol. 1999;163(3):1490-1497.

5. Smythies LE, Waites KB, Lindsey JR, Harris PR, Ghiara P, Smith PD. Helicobacter pylori-induced mucosal inflammation is Th1 mediated and exacerbated in IL-4, but not IFN-gamma, gene-deficient mice. J Immunol. 2000;165(2):1022-1029.

6. Tu S, et al. Overexpression of interleukin-1beta induces gastric inflammation and cancer and mobilizes myeloid-derived suppressor cells in mice. Cancer Cell. 2008;14(5):408-419.

7. Syu LJ, et al. Transgenic expression of interferon- $\gamma$ in mouse stomach leads to inflammation, metaplasia, and dysplasia. Am J Pathol. 2012;181(6):2114-2125.

8. O'Reilly LA, et al. Loss of NF- $\kappa \mathrm{B} 1$ causes gastric cancer with aberrant inflammation and expression of immune checkpoint regulators in a STAT-1-dependent manner. Immunity. 2018;48(3):570-583.e8.

9. Arisawa T, et al. Functional promoter polymorphisms of NFKB1 influence susceptibility to the diffuse type of gastric cancer. Oncol Rep. 2013;30(6):3013-3019.

10. El-Omar EM, et al. The role of interleukin-1 polymorphisms in the pathogenesis of gastric cancer. Nature. 2001;412(6842):99.

11. Oakley RH, Cidlowski JA. The biology of the glucocorticoid receptor: new signaling mechanisms in health and disease. J Allergy Clin Immunol. 2013;132(5):1033-1044.

12. Cain DW, Cidlowski JA. Immune regulation by glucocorticoids. Nat Rev Immunol. 2017;17(4):233-247.

13. Ford AC, et al. Glucocorticosteroid therapy in inflammatory bowel disease: systematic review and meta-analysis. Am J Gastroenterol.
2011;106(4):590-599; quiz 600.

14. Papierska L, Rabijewski M. Delay in diagnosis of adrenal insufficiency is a frequent cause of adrenal crisis. Int J Endocrinol. 2013;2013:482370.

15. Puar TH, Stikkelbroeck NM, Smans LC, Zelissen PM, Hermus AR. Adrenal crisis: still a deadly event in the 21st century. Am J Med. 2016;129(3):339.e1-339.e9.

16. Block TS, Murphy TI, Munster PN, Nguyen DP, Lynch FJ. Glucocorticoid receptor expression in 20 solid tumor types using immunohistochemistry assay. Cancer Manag Res. 2017;9:65-72.

17. Lien HC, Lu YS, Shun CT, Yao YT, Chang WC, Cheng AL. Differential expression of glucocorticoid receptor in carcinomas of the human digestive system. Histopathology. 2008;52(3):314-324.

18. Mills JC, Goldenring JR. Metaplasia in the stomach arises from gastric chief cells. Cell Mol Gastroenterol Hepatol. 2017;4(1):85-88.

19. Nam KT, et al. Mature chief cells are cryptic progenitors for metaplasia in the stomach. Gastroenterology. 2010;139(6):2028-2037.e9.

20. Engevik AC, et al. The development of spasmolytic polypeptide/TFF2-expressing metaplasia (SPEM) during gastric repair is absent in the aged stomach. Cell Mol Gastroenterol Hepatol. 2016;2(5):605-624.

21. Mills JC, Sansom OJ. Reserve stem cells: differentiated cells reprogram to fuel repair, metaplasia, and neoplasia in the adult gastrointestinal tract. Sci Signal. 2015;8(385):re8.

22. Wada T, et al. Functional role of CD $44 \mathrm{v}-\mathrm{xCT}$ system in the development of spasmolytic polypeptide-expressing metaplasia. Cancer Sci. 2013;104(10):1323-1329.

23. Bertaux-Skeirik N, et al. CD44 variant isoform 9 emerges in response to injury and contributes to the regeneration of the gastric epithelium. JPathol. 2017;242(4):463-475.

24. Willet SG, et al. Regenerative proliferation of differentiated cells by mTORC1-dependent paligenosis. EMBO J. 2018;37(7):e98311.

25. Lee HJ, et al. Gene expression profiling of metaplastic lineages identifies $\mathrm{CDH} 17$ as a prognostic marker in early stage gastric cancer. Gastroenterology. 2010;139(1):213-25.e3.

26. Weis VG, et al. Heterogeneity in mouse spasmolytic polypeptide-expressing metaplasia lineages identifies markers of metaplastic progression. Gut. 2013;62(9):1270-1279.

27. Isajevs $S$, et al. Different pattern of inflammatory and atrophic changes in the gastric mucosa of the greater and lesser curvature. J Gastrointestin Liver
Dis. 2015;24(4):429-434.

28. Ichinose M, et al. Proliferation, differentiation and morphogenesis of fetal rat glandular stomach transplanted under the kidney capsule of syngeneic hosts. Dev Growth Differ. 1997;39(5):635-642.

29. Zulian JG, Hosoya LY, Figueiredo PM, Ogias D, Osaki LH, Gama P. Corticosterone activity during early weaning reprograms molecular markers in rat gastric secretory cells. Sci Rep. 2017;7:45867.

30. Petersen CP, Mills JC, Goldenring JR. Murine models of gastric corpus preneoplasia. Cell Mol Gastroenterol Hepatol. 2017;3(1):11-26.

31. Mombaerts P, Iacomini J, Johnson RS, Herrup K, Tonegawa S, Papaioannou VE. RAG-1-deficient mice have no mature $\mathrm{B}$ and $\mathrm{T}$ lymphocytes. Cell. 1992;68(5):869-877.

32. Zuo L, Rothenberg ME. Gastrointestinal eosinophilia. Immunol Allergy Clin North Am. 2007;27(3):443-455.

33. Jeong S, et al. Distinct metaplastic and inflammatory phenotypes in autoimmune and adenocarcinoma-associated chronic atrophic gastritis. United European Gastroenterol J. 2017;5(1):37-44

34. Yu C, et al. Targeted deletion of a high-affinity GATA-binding site in the GATA-1 promoter leads to selective loss of the eosinophil lineage in vivo. JExp Med. 2002;195(11):1387-1395.

35. Petersen CP, et al. A signalling cascade of IL-33 to IL-13 regulates metaplasia in the mouse stomach. Gut. 2018;67(5):805-817.

36. Petersen CP, Weis VG, Nam KT, Sousa JF, Fingleton B, Goldenring JR. Macrophages promote progression of spasmolytic polypeptide-expressing metaplasia after acute loss of parietal cells. Gastroenterology. 2014;146(7):1727-38.e8.

37. Burclaff J, Osaki LH, Liu D, Goldenring JR, Mills JC. Targeted apoptosis of parietal cells is insufficient to induce metaplasia in stomach. Gastroenterology. 2017;152(4):762-766.e7.

38. Bhavsar PK, Sukkar MB, Khorasani N, Lee KY, Chung KF. Glucocorticoid suppression of CX3CL1 (fractalkine) by reduced gene promoter recruitment of NF-kappaB. FASEB J. 2008;22(6):1807-1816.

39. Dhawan L, Liu B, Blaxall BC, Taubman MB. A novel role for the glucocorticoid receptor in the regulation of monocyte chemoattractant protein-1 mRNA stability. J Biol Chem . 2007;282(14):10146-10152.

40. Poon M, Liu B, Taubman MB. Identification of a novel dexamethasone-sensitive RNA-destabilizing 
region on rat monocyte chemoattractant protein 1 mRNA. Mol Cell Biol. 1999;19(10):6471-6478.

41. Geissmann F, Jung S, Littman DR. Blood monocytes consist of two principal subsets with distinct migratory properties. Immunity. 2003;19(1):71-82.

42. Sunderkötter C, et al. Subpopulations of mouse blood monocytes differ in maturation stage and inflammatory response. J Immunol. 2004;172(7):4410-4417.

43. Imai $\mathrm{T}$, et al. Identification and molecular characterization of fractalkine receptor CX3CR1, which mediates both leukocyte migration and adhesion. Cell. 1997;91(4):521-530.

44. Kurihara T, Warr G, Loy J, Bravo R. Defects in macrophage recruitment and host defense in mice lacking the CCR2 chemokine receptor. J Exp Med.1997;186(10):1757-1762.

45. Biswas SK, Mantovani A. Macrophage plasticity and interaction with lymphocyte subsets: cancer as a paradigm. Nat Immunol.2010;11(10):889-896.

46. Hanna RN, et al. Patrolling monocytes control tumor metastasis to the lung. Science. 2015;350(6263):985-990.

47. Bhattacharyya S, Brown DE, Brewer JA, Vogt SK, Muglia LJ. Macrophage glucocorticoid receptors regulate Toll-like receptor 4-mediated inflammatory responses by selective inhibition of p38 MAP kinase. Blood. 2007;109(10):4313-4319.

48. Peek RM, Crabtree JE. Helicobacter infection and gastric neoplasia. J Pathol. 2006;208(2):233-248.

49. Correa P. Helicobacter pylori and gastric carcinogenesis. Am J Surg Pathol. 1995;19 Suppl 1:S37-S43.

50. Peek RM, et al. Heightened inflammatory response and cytokine expression in vivo to cagA+ Helicobacter pylori strains. Lab Invest. 1995;73(6):760-770.

51. Figueiredo C, et al. Helicobacter pylori and interleukin 1 genotyping: an opportunity to identify high-risk individuals for gastric carcinoma. J Natl Cancer Inst. 2002;94(22):1680-1687.

52. Amieva MR, El-Omar EM. Host-bacterial interactions in Helicobacter pylori infection. Gastroenterology. 2008;134(1):306-323.

53. El-Omar EM, et al. Increased risk of noncardia gastric cancer associated with proinflammatory cytokine gene polymorphisms. Gastroenterology. 2003;124(5):1193-1201.

54. Barnes PJ, Adcock IM. Glucocorticoid resistance in inflammatory diseases. Lancet. 2009;373(9678):1905-1917.

55. Correa P, Shiao YH. Phenotypic and genotypic events in gastric carcinogenesis. Cancer Res. 1994;54(7 Suppl):1941s-1943s.

56. Huh WJ, Khurana SS, Geahlen JH, Kohli K, Waller RA, Mills JC. Tamoxifen induces rapid, reversible atrophy, and metaplasia in mouse stomach. Gastroenterology. 2012;142(1):21-24.e7.

57. Hanna RN, et al. NR4A1 (Nur77) deletion polarizes macrophages toward an inflammatory phenotype and increases atherosclerosis. Circ Res. 2012;110(3):416-427.

58. Olingy CE, et al. Non-classical monocytes are biased progenitors of wound healing macrophages during soft tissue injury. Sci Rep. 2017;7(1):447.

59. Ridderstad Wollberg A, et al. Pharmacological inhibition of the chemokine receptor CX3CR1 attenuates disease in a chronic-relapsing rat model for multiple sclerosis. Proc Natl Acad Sci USA. 2014;111(14):5409-5414.

60. Poupel L, et al. Pharmacological inhibition of the chemokine receptor, CX3CR1, reduces atherosclerosis in mice. Arterioscler Thromb Vasc Biol. 2013;33(10):2297-2305.

61. Wakita H, Yanagawa T, Kuboi Y, Imai T. E6130, a novel CX3C chemokine receptor 1 (CX3CR1) modulator, attenuates mucosal inflammation and reduces CX3CR1+ leukocyte trafficking in mice with colitis. Mol Pharmacol. 2017;92(5):502-509.

62. Cruz-Topete D, Myers PH, Foley JF, Willis MS, Cidlowski JA. Corticosteroids are essential for maintaining cardiovascular function in male mice. Endocrinology. 2016;157(7):2759-2771. 